APPLIED MATHEMATICS AND MECHANICS (ENGLISH EDITION)

https://doi.org/10.1007/s10483-020-2668-8

\title{
Bifurcation and dynamic behavior analysis of a rotating cantilever plate in subsonic airflow*
}

\author{
Li MA, Minghui $\mathrm{YAO}^{\dagger}$, Wei ZHANG, Dongxing CAO \\ Beijing Key Laboratory of Nonlinear Vibrations and Strength of Mechanical Structures, \\ College of Mechanical Engineering, Beijing University of Technology, \\ Beijing 100124, China
}

(Received Apr. 10, 2020 / Revised Jul. 22, 2020)

\begin{abstract}
Turbo-machineries, as key components, have wide applications in civil, aerospace, and mechanical engineering. By calculating natural frequencies and dynamical deformations, we have explained the rationality of the series form for the aerodynamic force of the blade under the subsonic flow in our earlier studies. In this paper, the subsonic aerodynamic force obtained numerically is applied to the low pressure compressor blade with a low constant rotating speed. The blade is established as a pre-twist and presetting cantilever plate with a rectangular section under combined excitations, including the centrifugal force and the aerodynamic force. In view of the first-order shear deformation theory and von-Kármán nonlinear geometric relationship, the nonlinear partial differential dynamical equations for the warping cantilever blade are derived by Hamilton's principle. The second-order ordinary differential equations are acquired by the Galerkin approach. With consideration of 1:3 internal resonance and 1/2 sub-harmonic resonance, the averaged equation is derived by the asymptotic perturbation methodology. Bifurcation diagrams, phase portraits, waveforms, and power spectrums are numerically obtained to analyze the effects of the first harmonic of the aerodynamic force on nonlinear dynamical responses of the structure.
\end{abstract}

Key words subsonic aerodynamic force, asymptotic perturbation method, bifurcation and chaos

Chinese Library Classification O322

2010 Mathematics Subject Classification 34C28

\section{Introduction}

Blades, which are vital rotating structural units, are widely used in turbo-machinery of aeronautical and aerospace industries. It is well-known that blades are often exposed to harsh working conditions, especially for gas turbine blades. Vibration of blades under airflow excitation has been recognized as one of the major causes of system failure in the engineering field.

* Citation: MA, L., YAO, M. H., ZHANG, W., and CAO, D. X. Bifurcation and dynamic behavior analysis of a rotating cantilever plate in subsonic airflow. Applied Mathematics and Mechanics (English Edition), 41(12), 1861-1880 (2020) https://doi.org/10.1007/s10483-020-2668-8

$\dagger$ Corresponding author, E-mail: merry_mingming@163.com

Project supported by the National Natural Science Foundation of China (Nos. 11372015, 11832002, 11290152, 11427801, and 11972051)

(C)The Author(s) 2020 
Transverse vibrations of compressor blades often exhibit dynamic behaviors, which may lead to bifurcation phenomena. Dynamic behaviors of blades are very complex due to the coupling effect of fluid-structure interaction and strong nonlinear and unsteady characteristics. To design such structures properly, we need a profound understanding of their dynamic characteristics to avoid some undesirable disasters such as resonance phenomena and flutter.

Blade, a core component of modern rotating machine, endures the coupling effects of the centrifugal force and the aerodynamic load. In order to get a profound understanding of the vibration characteristics of the system, the establishment for a reasonable dynamical model is the most pressing concern for researchers. Plenty of theoretical analyses focus on the vibration characteristics of blades, which have been simplified as Euler-Bernoulli beams ${ }^{[1]}$ and Timoshenko beams ${ }^{[2-3]}$ under various conditions.

However, if the blade is thin, the aspect ratio is small, or higher frequencies and modes are needed, the model of the cantilever beam is highly inaccurate. Thus, the cantilever plate or the shell model is needed to describe the rotating blade. Sabuncu and Thomas ${ }^{[4]}$ numerically investigated the free vibration characteristics of the shrouded pre-twist rotating aerofoil blade without shear deformation and rotary inertia effects. Li and Zhang ${ }^{[5]}$ observed frequency loci veering phenomena rather than crossing in either rotating homogeneous plates or functionally graded (FG) plates. Mahi et al. ${ }^{[6]}$ and Bennoun et al. ${ }^{[7]}$ proposed a refined plate theory and a novel hyperbolic shear deformation theory to study the bending and free vibration of different kinds of composite plates. Xie et al. ${ }^{[8]}$ put forward a novel breathing model for the blade with cracks of the rotating shaft-disk-blade system subject to bending stresses and centrifugal stresses and compared the vibration responses of this model with two different crack models. Niu et al. ${ }^{[9]}$ studied free vibrations of a rotating blade, which is modeled as a pre-twisted FG cylindrical cantilever panel reinforced with graphene.

In addition to linear vibrations, some scholars have also studied the nonlinear oscillations of blades. Zhang and Zhao ${ }^{[10]}$ analyzed the nonlinear oscillations and amplitude-frequency characteristics for the composite laminated rotary plate enduring lateral and horizontal excitations. Chen et al. ${ }^{[11]}$ discussed the nonlinear steady-state responses of thin-walled structures in supercritical flow. Subrahmanyam et al. ${ }^{[12]}$ and Yao et al. ${ }^{[13]}$ studied the effects of the Coriolis terms and several other nonlinear terms on the oscillation and stability of the rotating thin-walled linearly pre-twist blade. Roy and Meguid ${ }^{[14]}$ found that the centrifugal force field decaying leads to a severe change in the dynamical responses of blades. Wang et al. ${ }^{[15]}$ explained Hopf bifurcation and saddle-node based on the reduced fluid-structure interaction model of the vortex-induced oscillations for turbine blades. The double Hopf bifurcations, global bifurcations, and multi-pulse chaotic dynamics of the laminated plate under certain internal resonances were investigated ${ }^{[16-17]}$. Zhang et al. ${ }^{[18-19]}$ studied sub-harmonic and superharmonic resonances combined with different resonance cases of a rotary blade under strong gas pressure. Steady-state responses, saturation, jump, and hysteresis are focused on. Ding et al. ${ }^{[20]}$ further illustrated the energy transfer between the saturation-like phenomena and the internal resonance modes. Liu et al. ${ }^{[21]}$ gave phase portraits, time history, Poincaré maps, and bifurcation diagrams with different parameters of an FG cylindrical shell with small initial geometric imperfect defects under complex excitations. Yao et al. ${ }^{[22]}$ explored the nonlinear transient dynamic responses and steady-state dynamic vibrations of the pre-twisted cylindrical shell model for the aero-engine compressor blade subject to the single point excitation and the uniform distribution excitation.

Enormous studies have been implemented about blades. However, few of them devoted to the nonlinear responses for the pre-twist and presetting rotating cantilever blade under subsonic airflow. The difficulty lies in the fact that we do not have an explicit expression for the blade in subsonic airflow. Therefore, in this paper, the explicit time dependent aerodynamic force expression of the blade in the subsonic airflow is introduced. The nonlinear dynamical behaviors of the blade subject to aerodynamic forces numerically obtained are investigated. Consider- 
ing the shear deformation and von-Kármán nonlinear geometric relationship, the equations of motion for the cantilever plate are derived by Hamilton's principle. The asymptotic perturbation methodology is introduced to deduce the averaged equation of the structure in case of 1:3 internal resonance and 1/2 sub-harmonic resonance. Bifurcation and chaotic dynamics of the system are analyzed numerically. Numerical simulation shows that the aerodynamic force has a significant effect on the periodic and chaotic motions of the rotating blade under specific cases. Since we can avoid chaotic motions of the system by changing the aerodynamic force, the large amplitude nonlinear vibration of the blade can be further controlled.

\section{Equations of motion}

The structure diagram of the rotary cantilever plate is shown in Fig. $1^{[23]}$. The blade is modeled as a presetting and pre-twist rotating cantilever plate with a rectangular section, which is clamped to a rigid hub with the radius $R$. The blade rotates with a constant rotating speed $\Omega_{0}$. The span length and the chord length of the blade are $L$ and $C$, respectively, where $L=R_{0}-R$. The thickness of the plate is marked as $h$. The pre-twist angle $\phi_{R}$ and the mounting angle $\theta_{r}$ exist at the free end and the fixed end of the plate, respectively. Three coordinate systems, i.e., the inertial coordinate system $(i, j, k)$, the rotating coordinate system $\left(e_{x}^{0}, e_{y}^{0}, e_{z}^{0}\right)$, and the sectional coordinate system $\left(e_{x}, e_{y}, e_{z}\right)$, are introduced, respectively. The origin of the inertial frame $(i, j, k)$ is the center of the hub. $\left(e_{x}^{0}, e_{y}^{0}, e_{z}^{0}\right)$ is located at the edge of the hub. $e_{x}^{0}, e_{y}^{0}$, and $e_{z}^{0}$ represent the spanwise direction, the chordwise direction, and the thickness direction, respectively.

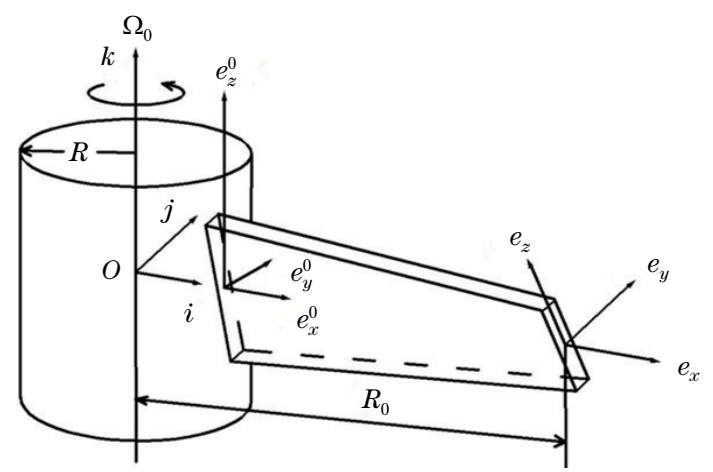

Fig. 1 Structure diagram of the rotating blade

Based on the research done by Yao et al. ${ }^{[23]}$, considering the first-order shear deformation and Kirchhoff hypothesis ${ }^{[24]}$, the dimensionless partial differential governing equations are expressed by the displacements of an arbitrary points on the neutral plane of the plate $u_{0}, v_{0}$, and $w_{0}$ along the $x^{-}, y$-, and $z$-directions. The nonlinear dynamic oscillations of the rotating blade are investigated qualitatively. The dimensionless parameters are introduced as follows:

$$
\left\{\begin{array}{l}
\bar{y}=\frac{y}{C}, \quad \bar{A}_{i j}=\frac{(L C)^{\frac{1}{2}}}{E h^{2}} A_{i j}, \quad \bar{B}_{i j}=\frac{(L C)^{\frac{1}{2}}}{E h^{3}} B_{i j}, \quad \bar{D}_{i j}=\frac{(L C)^{\frac{1}{2}}}{E h^{4}} D_{i j}, \\
\bar{I}_{i}=\frac{1}{\rho(L C)^{\frac{(1+i)}{2}}}, \quad \bar{\Omega}=\Omega \sqrt{\frac{\rho C L}{E}}, \quad \bar{t}=t \sqrt{\frac{E}{\rho C L}}, \quad \bar{\theta}=\theta \\
\bar{\theta}^{\prime}=L \theta^{\prime}, \quad \bar{r}=\frac{r}{L}, \quad \bar{C}_{\infty}=C_{\infty} \sqrt{\frac{\rho}{E}}, \quad \bar{p}=p \sqrt{\frac{\rho}{E}}, \quad \bar{\rho}_{\infty}=\frac{\rho_{\infty} L^{2}}{\rho A} .
\end{array}\right.
$$

The dimensionless nonlinear governing equations for the structure are described as follows: 
$\delta u_{0}$ :

$$
\begin{aligned}
& a_{10} \frac{\partial^{2} u_{0}}{\partial x^{2}}+a_{11} \frac{\partial^{2} u_{0}}{\partial y^{2}}+a_{12} \frac{\partial^{2} v_{0}}{\partial x \partial y}+a_{13} \frac{\partial w_{0}}{\partial x}+a_{14} \frac{\partial^{2} w_{0}}{\partial x^{2}}+a_{15} \frac{\partial w_{0}}{\partial x} \frac{\partial^{2} w_{0}}{\partial x^{2}} \\
& +a_{16} \frac{\partial w_{0}}{\partial x} \frac{\partial^{2} w_{0}}{\partial y^{2}}+a_{17} \frac{\partial w_{0}}{\partial y} \frac{\partial^{2} w_{0}}{\partial x \partial y}+a_{18} \frac{\partial^{2} \varphi_{x}}{\partial y^{2}}+a_{19} \frac{\partial^{2} \varphi_{x}}{\partial x^{2}}+a_{20} \frac{\partial^{2} \varphi_{y}}{\partial x \partial y} \\
= & a_{21} \ddot{u}_{0} \cos \Theta+a_{22} \ddot{v}_{0} \sin \Theta+a_{23} \ddot{w}_{0} \sin \Theta+a_{24} \dot{u}_{0} \sin \Theta \cdot \Omega_{0}+a_{25} \dot{u}_{0} \cos \Theta \cdot \Omega_{0} \\
& +a_{26} \dot{w}_{0} \cos \Theta \cdot \Omega_{0}+a_{27} x \cos \Theta \cdot \Omega_{0}^{2}+a_{28} \Omega_{0}^{2} \cos \Theta+a_{29} u_{0} \cdot \Omega_{0}^{2} \cos \Theta \\
& +a_{30} y \Omega_{0}^{2} \sin \Theta+a_{31} v_{0} \Omega_{0}^{2} \sin \Theta+a_{32} w_{0} \sin \Theta \cdot \Omega_{0}^{2}+a_{33} y \frac{\partial w_{0}}{\partial x} \cdot \Omega_{0}^{2} \\
& +a_{34} y \frac{\partial u_{0}}{\partial y} \cdot \Omega_{0}^{2}+a_{35}\left(\frac{C^{2}}{4}-y^{2}\right) \frac{\partial^{2} w_{0}}{\partial x^{2}} \cdot \Omega_{0}^{2}+a_{36}\left(\frac{C^{2}}{4}-y^{2}\right) \frac{\partial^{2} u_{0}}{\partial y^{2}} \cdot \Omega_{0}^{2} \\
& +a_{37} x \sin \Theta \cdot \dot{\Omega}_{0}+a_{38} \sin \Theta \cdot \dot{\Omega}_{0}+a_{39} u_{0} \sin \Theta \cdot \dot{\Omega}_{0}+a_{40} y \cos \Theta \cdot \dot{\Omega}_{0} \\
& +a_{41} v_{0} \cos \Theta \cdot \dot{\Omega}_{0}+a_{42} w_{0} \cos \Theta \cdot \dot{\Omega}_{0} .
\end{aligned}
$$

$\delta v_{0}:$

$$
\begin{aligned}
& b_{10} \frac{\partial^{2} v_{0}}{\partial x^{2}}+b_{11} \frac{\partial^{2} u_{0}}{\partial x \partial y}+b_{12} \frac{\partial^{2} v_{0}}{\partial y^{2}}+b_{13} \frac{\partial w_{0}}{\partial x} \frac{\partial^{2} w_{0}}{\partial x \partial y}+b_{14} \frac{\partial w_{0}}{\partial y} \frac{\partial^{2} w_{0}}{\partial y^{2}}+b_{15} \frac{\partial w_{0}}{\partial y} \frac{\partial^{2} w_{0}}{\partial x^{2}} \\
& +b_{16} \frac{\partial^{2} \varphi_{x}}{\partial x \partial y}+b_{17} \frac{\partial^{2} \varphi_{y}}{\partial x^{2}}+b_{18} \frac{\partial^{2} \varphi_{y}}{\partial y^{2}}+b_{19} \frac{\partial^{2} w_{0}}{\partial x \partial y}+b_{20} \frac{\partial w_{0}}{\partial y} \\
= & b_{21} \ddot{u}_{0} \sin \Theta+b_{22} \ddot{v}_{0} \cos \Theta+b_{23} \ddot{w}_{0} \cos \Theta+b_{24} \dot{u}_{0} \cos \Theta \cdot \Omega_{0}+b_{25} \dot{v}_{0} \sin \Theta \cdot \Omega_{0} \\
& +b_{26} \dot{w}_{0} \sin \Theta \cdot \Omega_{0}+b_{27} x \Omega_{0}^{2} \sin \Theta+b_{28} \Omega_{0}^{2} \sin \Theta+b_{29} \Omega_{0}^{2} \sin \Theta \cdot u_{0}+b_{30} y \Omega_{0}^{2} \cos \Theta \\
& +b_{31} v_{0} \cos \Theta \cdot \Omega_{0}^{2}+b_{32} w_{0} \cos \Theta \cdot \Omega_{0}^{2}+b_{33}(r+x) \frac{\partial w_{0}}{\partial x} \cdot \Omega_{0}^{2}+b_{34}(r+x) \frac{\partial v_{0}}{\partial x} \cdot \Omega_{0}^{2} \\
& +b_{35}\left(r(L-x)+\frac{1}{2}\left(L^{2}-x^{2}\right)\right) \frac{\partial^{2} w_{0}}{\partial x^{2}} \cdot \Omega_{0}^{2}+b_{36}\left(r(L-x)+\frac{1}{2}\left(L^{2}-x^{2}\right)\right) \frac{\partial^{2} v_{0}}{\partial x^{2}} \cdot \Omega_{0}^{2} \\
& +b_{37} x \cos \Theta \cdot \dot{\Omega}_{0}+b_{38} \cos \Theta \cdot \dot{\Omega}_{0}+b_{39} u_{0} \cos \Theta \cdot \dot{\Omega}_{0}+b_{40} y \sin \Theta \cdot \dot{\Omega}_{0} \\
& +b_{41} v_{0} \sin \Theta \cdot \dot{\Omega}_{0}+b_{42} w_{0} \sin \Theta \cdot \dot{\Omega}_{0} .
\end{aligned}
$$


$\delta w_{0}:$

$$
\begin{aligned}
& c_{10}\left(\frac{\partial w_{0}}{\partial x}\right)^{2} \frac{\partial^{2} w_{0}}{\partial x^{2}}+c_{11}\left(\frac{\partial w_{0}}{\partial x}\right)^{2} \frac{\partial^{2} w_{0}}{\partial y^{2}}+c_{12} \frac{\partial w_{0}}{\partial x} \frac{\partial w_{0}}{\partial y} \frac{\partial^{2} w_{0}}{\partial x \partial y}+c_{13} \frac{\partial w_{0}}{\partial x} \frac{\partial^{2} w_{0}}{\partial x^{2}} \\
& +c_{14}\left(\frac{\partial w_{0}}{\partial y}\right)^{2} \frac{\partial^{2} w_{0}}{\partial y^{2}}+c_{15}\left(\frac{\partial w_{0}}{\partial y}\right)^{2} \frac{\partial^{2} w_{0}}{\partial x^{2}}+c_{16} \frac{\partial^{2} w_{0}}{\partial y \partial x} \frac{\partial w_{0}}{\partial y}+c_{17} \frac{\partial^{2} u_{0}}{\partial x^{2}} \frac{\partial w_{0}}{\partial x}+c_{18} \frac{\partial^{2} u_{0}}{\partial y^{2}} \frac{\partial w_{0}}{\partial x} \\
& +c_{19} \frac{\partial^{2} v_{0}}{\partial x \partial y} \frac{\partial w_{0}}{\partial x}+c_{20}\left(\frac{\partial w_{0}}{\partial x}\right)^{2}+c_{21} \frac{\partial^{2} \varphi_{x}}{\partial y^{2}} \frac{\partial w_{0}}{\partial x}+c_{22} \frac{\partial^{2} \varphi_{x}}{\partial x^{2}} \frac{\partial w_{0}}{\partial x}+c_{23} \frac{\partial^{2} \varphi_{y}}{\partial x \partial y} \frac{\partial w_{0}}{\partial x} \\
& +c_{24} \frac{\partial^{2} u_{0}}{\partial x \partial y} \frac{\partial w_{0}}{\partial y}+c_{25} \frac{\partial^{2} v_{0}}{\partial x^{2}} \frac{\partial w_{0}}{\partial y}+c_{26} \frac{\partial^{2} v_{0}}{\partial y^{2}} \frac{\partial w_{0}}{\partial y}+c_{27} \frac{\partial^{2} \varphi_{x}}{\partial x \partial y} \frac{\partial w_{0}}{\partial y}+c_{28} \frac{\partial^{2} \varphi_{y}}{\partial x^{2}} \frac{\partial w_{0}}{\partial y} \\
& +c_{29} \frac{\partial^{2} \varphi_{y}}{\partial y^{2}} \frac{\partial w_{0}}{\partial y}+c_{30}\left(\frac{\partial w_{0}}{\partial y}\right)^{2}+c_{31} \frac{\partial u_{0}}{\partial y} \frac{\partial^{2} w_{0}}{\partial x \partial y}+c_{32} \frac{\partial^{2} v_{0}}{\partial x} \frac{\partial^{2} w_{0}}{\partial x \partial y}+c_{33} \frac{\partial \varphi_{x}}{\partial y} \frac{\partial^{2} w_{0}}{\partial x \partial y} \\
& +c_{34} \frac{\partial \varphi_{y}}{\partial x} \frac{\partial^{2} w_{0}}{\partial x \partial y}+c_{35} \frac{\partial u_{0}}{\partial x} \frac{\partial^{2} w_{0}}{\partial x^{2}}+c_{36} \frac{\partial v_{0}}{\partial y} \frac{\partial^{2} w_{0}}{\partial x^{2}}+c_{37} \frac{\partial \varphi_{x}}{\partial x} \frac{\partial^{2} w_{0}}{\partial x^{2}}+c_{38} w_{0} \frac{\partial^{2} w_{0}}{\partial x^{2}} \\
& +c_{39} \frac{\partial^{2} \varphi_{y}}{\partial y} \frac{\partial^{2} w_{0}}{\partial x^{2}}+c_{40} \frac{\partial u_{0}}{\partial x} \frac{\partial^{2} w_{0}}{\partial y^{2}}+c_{41} \frac{\partial v_{0}}{\partial y} \frac{\partial^{2} w_{0}}{\partial y^{2}}+c_{42} \frac{\partial \varphi_{x}}{\partial x} \frac{\partial^{2} w_{0}}{\partial y^{2}}+c_{43} w_{0} \frac{\partial^{2} w_{0}}{\partial y^{2}} \\
& +c_{44} \frac{\partial w_{0}}{\partial x} \frac{\partial^{2} w_{0}}{\partial y^{2}}+c_{45} \frac{\partial \varphi_{y}}{\partial y} \frac{\partial^{2} w_{0}}{\partial y^{2}}+c_{46} \frac{\partial^{2} u_{0}}{\partial x^{2}}+c_{47} \frac{\partial^{2} v_{0}}{\partial x \partial y}+c_{48} \frac{\partial^{2} \varphi_{x}}{\partial x^{2}}+c_{49} \frac{\partial^{2} w_{0}}{\partial x} \\
& +c_{50} \frac{\partial^{2} u_{0}}{\partial x^{2}}+c_{51} \frac{\partial^{2} \varphi_{y}}{\partial x \partial y}+c_{52} \frac{\partial^{2} w_{0}}{\partial x^{2}}+c_{53} \frac{\partial \varphi_{x}}{\partial x}+c_{54} \frac{\partial^{2} w_{0}}{\partial y^{2}}+c_{55} \frac{\partial \varphi_{y}}{\partial y}+c_{56} \\
& +c_{57} \sin (\Omega t)+c_{59} \sin (2 \Omega t)+c_{58} \cos (\Omega t)+c_{60} \cos (2 \Omega t) \\
& =c_{61}\left(r(L-x)+\frac{1}{2}\left(L^{2}-x^{2}\right)\right) \frac{\partial w_{0}}{\partial x} \cdot \Omega_{0}^{2}+c_{62}\left(r(L-x)+\frac{1}{2}\left(L^{2}-x^{2}\right)\right) \frac{\partial u_{0}}{\partial y} \cdot \Omega_{0}^{2} \\
& +c_{63}(r+x) \frac{\partial^{2} w_{0}}{\partial x^{2}} \cdot \Omega_{0}^{2}+c_{64}(r+x) \frac{\partial^{2} u_{0}}{\partial y^{2}} \cdot \Omega_{0}^{2}+c_{65} y \frac{\partial w_{0}}{\partial x} \cdot \Omega_{0}^{2}+c_{66} y \frac{\partial v_{0}}{\partial x} \cdot \Omega_{0}^{2} \\
& +c_{67}\left(\frac{C^{2}}{4}-y^{2}\right) \frac{\partial^{2} w_{0}}{\partial x^{2}} \cdot \Omega_{0}^{2}+c_{68}\left(\frac{C^{2}}{4}-y^{2}\right) \frac{\partial^{2} v_{0}}{\partial x^{2}} \cdot \Omega_{0}^{2}+c_{69} \ddot{v}_{0} \sin \theta+c_{70} \ddot{w}_{0} \\
& +c_{71} x \ddot{\varphi}_{x} \cos \Theta+c_{72} x^{2} \ddot{w}_{0} \cos \Theta+c_{73} x \ddot{\varphi}_{y} \sin \Theta+c_{74} x \dot{\varphi}_{x} \Omega_{0}+c_{75} x \dot{w}_{0} \Omega_{0} \\
& +c_{76} x \dot{\varphi}_{y} \Omega_{0} \cos \Theta+c_{77} x \varphi_{x} \Omega_{0}^{2} \cos \Theta+c_{78} x w_{0} \Omega_{0}^{2} \cos \Theta+c_{79} x \varphi_{y} \Omega_{0}^{2} \sin \Theta \\
& +c_{80} x \Omega_{0}^{2} \sin \Theta+c_{81} x \varphi_{x} \dot{\Omega}_{0} \sin \Theta+c_{82} x^{2} w_{0} \dot{\Omega}_{0} \sin \Theta+c_{83} x \varphi_{y} \dot{\Omega}_{0} \cos \Theta \\
& +c_{84} x \dot{\Omega}_{0} \cos \Theta
\end{aligned}
$$




$$
\begin{aligned}
& \delta \varphi_{x} \\
& d_{10} \frac{\partial^{2} u_{0}}{\partial x^{2}}+d_{11} \frac{\partial^{2} u_{0}}{\partial y^{2}}+d_{12} \frac{\partial^{2} v_{0}}{\partial x \partial y}+d_{13} \frac{\partial w_{0}}{\partial x} \frac{\partial^{2} w_{0}}{\partial x^{2}}+d_{14} \frac{\partial w_{0}}{\partial y} \frac{\partial^{2} w_{0}}{\partial x \partial y}+d_{15} \frac{\partial w_{0}}{\partial x} \frac{\partial^{2} w_{0}}{\partial y^{2}} \\
& +d_{16} \frac{\partial w_{0}}{\partial x}+d_{17} \frac{\partial^{2} w_{0}}{\partial x^{2}}+d_{18} \frac{\partial^{2} \varphi_{x}}{\partial x^{2}}+d_{19} \frac{\partial^{2} \varphi_{x}}{\partial y^{2}}+d_{20} \frac{\partial^{2} \varphi_{y}}{\partial x \partial y}+d_{21} \frac{\partial w_{0}}{\partial x}+d_{22} \varphi_{x} \\
& =d_{23} \ddot{\varphi}_{x} \cos \Theta+d_{24} x \ddot{w}_{0} \cos \Theta+d_{25} \ddot{\varphi}_{y} \sin \Theta+d_{26} \dot{\varphi}_{x} \cdot \Omega_{0}+d_{27} x \dot{w}_{0} \cdot \Omega_{0}+d_{28} \dot{\varphi}_{y} \cos \Theta \cdot \Omega_{0} \\
& +d_{29} \varphi_{x} \cos \Theta \cdot \Omega_{0}^{2}+d_{30} x w_{0} \cos \Theta \cdot \Omega_{0}^{2}+d_{31} \varphi_{y} \sin \Theta \cdot \Omega_{0}^{2}+d_{32} \sin \Theta \cdot \Omega_{0}^{2} \\
& +d_{33} \varphi_{x} \sin \Theta \cdot \dot{\Omega}_{0}+d_{34} x w_{0} \sin \Theta \cdot \dot{\Omega}_{0}+d_{35} \varphi_{y} \cos \Theta \cdot \dot{\Omega}_{0}+d_{36} \cos \Theta \cdot \dot{\Omega}_{0} . \\
& \delta \varphi_{y} \\
& e_{10} \frac{\partial^{2} v_{0}}{\partial x^{2}}+e_{11} \frac{\partial^{2} u_{0}}{\partial x \partial y}+e_{12} \frac{\partial^{2} v_{0}}{\partial y^{2}}+e_{13} \frac{\partial w_{0}}{\partial y} \frac{\partial^{2} w_{0}}{\partial x^{2}}+e_{14} \frac{\partial w_{0}}{\partial x} \frac{\partial^{2} w_{0}}{\partial x \partial y}+e_{15} \frac{\partial w_{0}}{\partial y} \frac{\partial^{2} w_{0}}{\partial y^{2}} \\
& +e_{16} \frac{\partial^{2} w_{0}}{\partial x \partial y}+e_{17} \frac{\partial^{2} \varphi_{x}}{\partial x \partial y}+e_{18} \frac{\partial^{2} \varphi_{y}}{\partial x^{2}}+e_{19} \frac{\partial^{2} \varphi_{y}}{\partial y^{2}}+e_{20} \frac{\partial w_{0}}{\partial y}+e_{21} \varphi_{y} \\
& =e_{22} \ddot{\varphi}_{x} \sin \Theta+e_{23} x \ddot{w}_{0} \sin \Theta+e_{24} \ddot{\varphi}_{y} \cos \Theta+e_{25} \dot{\varphi}_{x} \cos \Theta \cdot \Omega_{0}+e_{26} x \dot{w}_{0} \cos \Theta \cdot \Omega_{0} \\
& +e_{27} \dot{\varphi}_{y} \sin \Theta \cdot \Omega_{0}+e_{28} \varphi_{x} \sin \Theta \cdot \Omega_{0}^{2}+e_{29} x w_{0} \sin \Theta \cdot \Omega_{0}^{2}+e_{30} \varphi_{y} \cos \Theta \cdot \Omega_{0}^{2} \\
& +e_{31} \cos \Theta \cdot \Omega_{0}^{2}+e_{32} \varphi_{x} \cos \Theta \cdot \dot{\Omega}_{0}+e_{33} x w_{0} \cos \Theta \cdot \dot{\Omega}_{0}+e_{34} \varphi_{y} \sin \Theta \cdot \dot{\Omega}_{0} \\
& +e_{35} \sin \Theta \cdot \dot{\Omega}_{0}
\end{aligned}
$$

Here, $\theta$ is the twist angle of the plate, which is defined as $\theta=\theta_{r}+\left(\theta_{R} / L\right) \cdot x ; \varphi_{x}$ denotes the rotation of a transverse normal about the $y$-axis, and $\varphi_{y}$ denotes the rotation about the $x$-axis, where $\varphi_{x}=-\frac{\partial w_{0}}{\partial x}+\gamma_{x z}$, and $\varphi_{y}=-\frac{\partial w_{0}}{\partial y}+\gamma_{y z} ; \Theta$ is the rotary angle of the plate, which can be written as $\Theta=\Omega_{0} \cdot t$. These parameters can be found in Ref. [23].

The Galerkin method, which is derived by the Taylor expansion approach, is proposed for transferring a continuous operator problem to a discrete one. It is a convergent method in mathematics. This method may provide useful qualitative discussion of nonlinear behaviors for a dynamical system. The second-order ordinary differential dimensionless equations for the system are derived by applying the Galerkin method given by Ding et al. ${ }^{[25]}$. In this study, only the first two order modes of the later vibration are considered. The lateral displacement $w(x, y, t)$ and the angular displacement functions $\varphi_{x}(x, y, t)$ and $\varphi_{y}(x, y, t)$ can be found in Eq. (26) of Ref. [23]. The in-plane displacements are expressed as follows:

$$
\left\{\begin{array}{l}
u(x, y, t)=u_{1}(t) \sin \frac{\pi x}{2 L} \cos \frac{\pi y}{C}+u_{2}(t) \sin \frac{3 \pi x}{2 L} \cos \frac{2 \pi y}{C} \\
v(x, y, t)=v_{1}(t) \sin \frac{\pi x}{2 L} \sin \frac{\pi y}{C}+v_{2}(t) \sin \frac{3 \pi x}{2 L} \sin \frac{2 \pi y}{C}
\end{array}\right.
$$

where $u, v$, and $w$ are the displacements of an arbitrary point on the plate along the $x^{-}, y^{-}$, and $z$-directions, respectively. 
The transverse ordinary differential dimensionless equations of the rotating structure are deduced by using the Galerkin method as follows:

$$
\begin{aligned}
& \ddot{w}_{1}+\left(\alpha_{1}+f_{11} \cos \left(\Omega_{0} t\right)+f_{12} \sin \left(\Omega_{0} t\right)\right) w_{1}+\alpha_{2} \dot{w}_{1}+\alpha_{3} w_{1}^{2}+\alpha_{4} w_{1} w_{2}+\alpha_{5} w_{1} w_{2}^{2}+\alpha_{6} w_{1}^{2} w_{2} \\
& +\alpha_{7} w_{1}^{3}+\alpha_{8} w_{2}^{2}+\alpha_{9} w_{2}^{3}+\left(\alpha_{10}+f_{13} \cos \left(\Omega_{0} t\right)+f_{14} \sin \left(\Omega_{0} t\right)\right) w_{2}+\alpha_{11} \dot{w}_{2}+\alpha_{12} \ddot{w}_{2} \\
= & \alpha_{13}+f_{15} \cos \left(\Omega_{0} t\right)+f_{16} \sin \left(\Omega_{0} t\right)+g_{1}\left(f_{2} \sin \left(\Omega_{1} t\right)+f_{1} \cos \left(\Omega_{1} t\right)\right. \\
& \left.+f_{4} \sin \left(2 \Omega_{1} t\right)+f_{3} \cos \left(2 \Omega_{1} t\right)\right) \\
& \ddot{w}_{2}+\left(\beta_{1}+f_{21} \cos \left(\Omega_{0} t\right)+f_{22} \sin \left(\Omega_{0} t\right)\right) w_{2}+\beta_{2} \dot{w}_{2}+\beta_{3} w_{2}^{2}+\beta_{4} w_{2} w_{1}+\beta_{5} w_{2} w_{1}^{2}+\beta_{6} w_{2}^{2} w_{1} \\
& +\beta_{7} w_{2}^{3}+\beta_{8} w_{1}^{2}+\beta_{9} w_{1}^{3}+\left(\beta_{10}+f_{23} \cos \left(\Omega_{0} t\right)+f_{24} \sin \left(\Omega_{0} t\right)\right) w_{1}+\beta_{11} \dot{w}_{1}+\beta_{12} \ddot{w}_{1} \\
= & \beta_{13}+f_{25} \cos \left(\Omega_{0} t\right)+f_{26} \sin \left(\Omega_{0} t\right)+g_{2}\left(f_{2} \sin \left(\Omega_{1} t\right)+f_{1} \cos \left(\Omega_{1} t\right)+f_{4} \sin \left(2 \Omega_{1} t\right)\right. \\
& \left.+f_{3} \cos \left(2 \Omega_{1} t\right)\right),
\end{aligned}
$$

where $\Omega_{1}$ is the frequency of the aerodynamic force ${ }^{[23]}$.

\section{Perturbation analysis}

The perturbation method, which is called the small parameter expansion method, aims to find an asymptotic solution to a problem, especially a nonlinear problem. In Eq. (5), the square and cubic nonlinear terms are presented. Thus, it is reasonable to exploit the asymptotic perturbation methodology ${ }^{[26]}$ to derive the average equations, which guarantees the accuracy of the solution to a certain extent.

Considering 1/2 sub-harmonic resonance and 1:3 internal resonance, the relationship between $\omega_{1}$ and $\omega_{2}$ for the structure is written as follows:

$$
3 \omega_{1}=\omega_{2}, \quad \omega_{1}=\frac{1}{2} \Omega+\varepsilon^{2} \sigma_{1}, \quad \omega_{2}=\frac{3}{2} \Omega+\varepsilon^{2} \sigma_{2},
$$

where $\sigma_{1}$ and $\sigma_{2}$ are two detuning parameters, and $\Omega$ is the frequency of the external excitation.

By introducing the following time scale transformation, the positive rational number $q$ is constant in the following deduction:

$$
\tau=\varepsilon^{q} t
$$

The power series solutions of the equations for the blade under $1 / 2$ sub-harmonic resonance and 1:3 internal resonance are written as follows:

$$
\begin{aligned}
& x(t)=\sum_{n=-\infty}^{\infty} \varepsilon^{r_{n}} \psi_{n}(\tau, \varepsilon) \mathrm{e}^{-\mathrm{i} n \frac{\Omega}{2} t}, \\
& y(t)=\sum_{n=-\infty}^{\infty} \varepsilon^{r_{n}} \phi_{n}(\tau, \varepsilon) \mathrm{e}^{-\mathrm{i} n \frac{3 \Omega}{2} t} .
\end{aligned}
$$

In the above expression, if $n \neq 0$, then $r_{n}=|n|$, otherwise $r_{0}=r$. In the following derivation, we assume that the value of $r_{n}$ does not change. 
Functions $\psi_{n}(\tau, \varepsilon)$ and $\phi_{n}(\tau, \varepsilon)$ are given by

$$
\begin{aligned}
\psi_{n}(\tau, \varepsilon) & =\sum_{i=0}^{+\infty} \varepsilon^{\mathrm{i}} \psi_{n}^{(\mathrm{i})}(\tau), \\
\phi_{n}(\tau, \varepsilon) & =\sum_{i=0}^{+\infty} \varepsilon^{\mathrm{i}} \phi_{n}^{(\mathrm{i})}(\tau) .
\end{aligned}
$$

Suppose when $\varepsilon \rightarrow 0$, the limits of $\psi_{n}(\tau, \varepsilon)$ and $\phi_{n}(\tau, \varepsilon)$ exist. Meanwhile, we define $\psi_{n}^{(0)}=$ $\psi_{n}, \phi_{n}^{(0)}=\phi_{n}$ for $n \neq 1$ and $\psi_{1}^{(0)}=\psi, \phi_{1}^{(0)}=\phi$ for $n=1$. When $n=2$, we obtain the following derivative form ${ }^{[26]}$ :

$$
\begin{aligned}
& \frac{\mathrm{d}}{\mathrm{d} t}\left(\psi_{n} \mathrm{e}^{-\mathrm{i} n \frac{\Omega}{2} t}\right)=\left(-\mathrm{i} n \frac{\Omega}{2} \psi_{n}+\varepsilon^{q} \frac{\mathrm{d} \psi_{n}}{\mathrm{~d} \tau}\right) \mathrm{e}^{-\mathrm{i} n \frac{\Omega}{2} t}, \\
& \frac{\mathrm{d}}{\mathrm{d} t}\left(\phi_{n} \mathrm{e}^{-\mathrm{i} n \frac{3 \Omega}{2} t}\right)=\left(-\mathrm{i} n \frac{3}{2} \Omega \phi_{n}+\varepsilon^{q} \frac{\mathrm{d} \phi_{n}}{\mathrm{~d} \tau}\right) \mathrm{e}^{-\mathrm{i} n \frac{3 \Omega}{2} t} .
\end{aligned}
$$

To solve the coefficients $\psi_{n}(\tau, \varepsilon)$ and $\phi_{n}(\tau, \varepsilon)$, substitute the scale transformation, Eq. (5), and Eq. (7) into Eq. (4). Then, the equations for each $n$ order harmonic and certain orders of approximate values on the perturbation parameter $\varepsilon$ are derived as follows.

When $n=0$ and $r=2$, the following expressions are obtained:

$$
\begin{aligned}
\psi_{0}= & -\frac{4}{\Omega^{2}}\left(2 \alpha_{3} \psi_{1} \psi_{1}^{*}-2 f_{4} \sin \left(\Omega_{1} t\right) \cos \left(\Omega_{1} t\right)+2 \alpha_{8} \phi_{1} \phi_{1}^{*}-\alpha_{20} \sin \left(\Omega_{0} t\right)\right. \\
& \left.-\alpha_{19} \cos \left(\Omega_{0} t\right)-2 g_{1} f_{3} \cos ^{2}\left(\Omega_{1} t\right)-\alpha_{18}+g_{1} f_{3}\right), \\
\phi_{0}= & -\frac{4}{9 \Omega^{2}}\left(2 \beta_{3} \phi_{1} \phi_{1}^{*}-2 f_{14} \sin \left(\Omega_{1} t\right) \cos \left(\Omega_{1} t\right)+2 \beta_{8} \psi_{1} \psi_{1}^{*}+f_{13}\right. \\
& \left.-\beta_{19} \cos \left(\Omega_{0} t\right)-\beta_{20} \sin \left(\Omega_{0} t\right)-2 f_{13} \cos ^{2}\left(\Omega_{1} t\right)\right),
\end{aligned}
$$

where the items with asterisk are the complex conjugate of the corresponding items.

When $n=2$, considering the derivative form of Eq. (9) yields

$$
\begin{aligned}
\psi_{2} & =-\frac{2}{3} \frac{g_{1} f_{1}-2 \alpha_{3} \psi_{1}^{2}+I g_{1} f_{2}-2 \alpha_{4} \psi_{1}^{*} \phi_{1}}{\Omega^{2}}, \\
\phi_{2} & =\frac{4}{27} \frac{\beta_{3} \phi_{1}^{2}}{\Omega^{2}} .
\end{aligned}
$$

When $n=1$ and $q=2$, the equations can be established. We obtain the following equations:

$$
\begin{aligned}
& -I \Omega D_{1}\left(\psi_{1}\right)+2 \alpha_{3} \psi_{0} \psi_{1}+\alpha_{4} \phi_{0} \psi_{1}-\frac{1}{2} I \alpha_{2} \psi_{1} \Omega+\Omega \sigma_{1} \psi_{1}+2 \alpha_{3} \psi_{2} \psi_{1}^{*} \\
& +3 \alpha_{7} \psi_{1}^{2} \psi_{1}^{*}+\alpha_{4} \phi_{1} \psi_{2}^{*}+\alpha_{6} \psi_{1}^{*} \phi_{1}+2 \alpha_{5} \psi_{1} \phi_{1} \phi_{1}^{*}=0 \\
& -3 I \Omega D_{1}\left(\phi_{1}\right)+2 \beta_{3} \phi_{0} \phi_{1}+\beta_{4} \phi_{1} \psi_{0}+2 \beta_{3} \phi_{2} \phi_{1}^{*}+3 \beta_{7} \phi_{1}^{2} \phi_{1}^{*}+2 \beta_{8} \psi_{1} \psi_{2} \\
& +3 \Omega \sigma_{2} \phi_{1}+2 \beta_{5} \phi_{1} \psi_{1} \psi_{1}^{*}+\beta_{9} \psi_{1}^{3}-\frac{3}{2} I \beta_{14} \phi_{1} \Omega-\frac{3}{2} I \beta_{2} \phi_{1} \Omega=0 .
\end{aligned}
$$

Substitute Eqs. (10) and (11) into Eq. (12). Then, the differential equations about $\psi_{1}$ and $\phi_{1}$ are obtained as follows:

$$
\begin{aligned}
D_{1}\left(\psi_{1}\right)= & \left(h_{1} f_{2}+I h_{2} f_{1}\right) \phi_{1}+\left(I h_{3}-I \sigma_{1}+h_{4}\right) \psi_{1}+\left(h_{5} f_{2}+I h_{6} f_{1}\right) \psi_{1}^{*} \\
& +I h_{7} \phi_{1} \phi_{1}^{*} \psi_{1}+I h_{8} \psi_{1}^{*} \psi_{1}^{2}+I h_{9} \phi_{1} \psi_{1}^{* 2}, \\
D_{1}\left(\phi_{1}\right)= & \left(I k_{1}-I \sigma_{2}+k_{2}\right) \phi_{1}+\left(k_{3} f_{2}+I k_{4} f_{1}\right) \psi_{1}+I k_{5} \phi_{1} \psi_{1} \psi_{1}^{* 2}+I k_{6} \phi_{1}^{*} \phi_{1}^{2}+I k_{7} \psi_{1}^{3} .
\end{aligned}
$$


Let $\psi_{1}$ and $\phi_{1}$ be in the following forms:

$$
\begin{aligned}
& \psi_{1}=\frac{1}{2} a_{1} \mathrm{e}^{\mathrm{i} \varphi_{1}}, \\
& \phi_{1}=\frac{1}{2} a_{2} \mathrm{e}^{\mathrm{i} \varphi_{2}} .
\end{aligned}
$$

The following polar coordinate averaged equation is acquired by extraction of the real and imaginary parts after substituting Eq. (14) into Eq. (13):

$$
\begin{aligned}
\dot{a}_{1}= & h_{4} a_{1}-h_{5} f_{2} a_{1}+\cos \left(\varphi_{1}-\varphi_{2}\right) a_{2} f_{2} h_{1}+\sin \left(\varphi_{1}-\varphi_{2}\right) a_{2} f_{1} h_{2} \\
+ & 4 a_{1}^{2} \cos ^{2} \varphi_{1} \sin \left(\varphi_{1}-\varphi_{2}\right) a_{2} h_{9}-a_{1}^{2} \sin \left(\varphi_{1}-\varphi_{2}\right) a_{2} h_{9}+2 a_{1}^{2} \sin \varphi_{2} \cos \varphi_{1} a_{2} h_{9} \\
+ & \sin \left(2 \varphi_{1}\right) a_{1} f_{1} h_{6}+2\left(\cos \varphi_{1}\right)^{2} a_{1} f_{2} h_{5}, \\
a_{1} \dot{\varphi}_{1}= & h_{8} a_{1}^{3}+h_{7} a_{2}^{2}-a_{1} \sigma_{1}+a_{1} h_{3}-\sin \left(\varphi_{1}-\varphi_{2}\right) a_{2} f_{2} h_{1}-\sin \left(2 \varphi_{1}\right) a_{1} f_{2} h_{5} \\
& -a_{1}^{2} \cos \left(\varphi_{1}-\varphi_{2}\right) a_{2} h_{9}-2 a_{1}^{2} \cos \varphi_{2} \cos \varphi_{1} a_{2} h_{9}+4 a_{1}^{2} \cos ^{2} \varphi_{1} \cos \left(\varphi_{1}-\varphi_{2}\right) a_{2} h_{9} \\
& +\cos \left(\varphi_{1}-\varphi_{2}\right) a_{2} f_{1} h_{2}+\cos \left(2 \varphi_{1}\right) h_{6} a_{1} f_{1}, \\
& \\
\dot{a}_{2}=- & 4 a_{1}^{3} \cos ^{2} \varphi_{1} \sin \left(\varphi_{1}-\varphi_{2}\right) k_{7}+a_{1}^{3} \sin \left(\varphi_{1}-\varphi_{2}\right) k_{7}-2 a_{1}^{3} k_{7} \sin \varphi_{2} \cos \varphi_{1} \\
- & \sin \left(\varphi_{1}-\varphi_{2}\right) k_{4} a_{1} f_{1}+\cos \left(\varphi_{1}-\varphi_{2}\right) k_{3} a_{1} f_{2}+k_{2} a_{2}, \\
& \\
a_{2} \dot{\varphi}_{2}= & k_{6} a_{2}^{3}+\sin \left(\varphi_{1}-\varphi_{2}\right) k_{3} a_{1} f_{2}+\cos \left(\varphi_{1}-\varphi_{2}\right) k_{4} a_{1} f_{1}-a_{1}^{3} \cos \left(\varphi_{1}-\varphi_{2}\right) k_{7} \\
& -2 a_{1}^{3} k_{7} \cos \varphi_{2} \cos \varphi_{1}+4 a_{1}^{3} \cos ^{2} \varphi_{1} \cos \left(\varphi_{1}-\varphi_{2}\right) k_{7}+k_{1} a_{2}-a_{2} \sigma_{2}+k_{5} a_{1}^{2} .
\end{aligned}
$$

When $\dot{a}_{1}=\dot{a}_{2}=\dot{\varphi}_{1}=\dot{\varphi}_{2}=0$, the following equations of the steady-state motions of the system are obtained:

$$
\begin{aligned}
& \left(h_{5} f_{2} a_{1}-h_{4} a_{1}\right)^{2}+\left(h_{8} a_{1}^{3}+h_{7} a_{2}^{2}-a_{1} \sigma_{1}+a_{1} h_{3}\right)^{2} \\
= & \left(\cos \left(\varphi_{1}-\varphi_{2}\right) a_{2} f_{2} h_{1}+\sin \left(\varphi_{1}-\varphi_{2}\right) a_{2} f_{1} h_{2}+4 a_{1}^{2} \cos ^{2} \varphi_{1} \sin \left(\varphi_{1}-\varphi_{2}\right) a_{2} h_{9}\right. \\
& -a_{1}^{2} \sin \left(\varphi_{1}-\varphi_{2}\right) a_{2} h_{9}+2 a_{1}^{2} \sin \varphi_{2} \cos \varphi_{1} a_{2} h_{9}+\sin \left(2 \varphi_{1}\right) a_{1} f_{1} h_{6} \\
& \left.+2\left(\cos \varphi_{1}\right)^{2} a_{1} f_{2} h_{5}\right)^{2}+\left(\sin \left(\varphi_{1}-\varphi_{2}\right) a_{2} f_{2} h_{1}+\sin \left(2 \varphi_{1}\right) a_{1} f_{2} h_{5}+a_{1}^{2} \cos \left(\varphi_{1}-\varphi_{2}\right) a_{2} h_{9}\right. \\
& +2 a_{1}^{2} \cos \varphi_{2} \cos \varphi_{1} a_{2} h_{9}-4 a_{1}^{2} \cos ^{2} \varphi_{1} \cos \left(\varphi_{1}-\varphi_{2}\right) a_{2} h_{9}-\cos \left(\varphi_{1}-\varphi_{2}\right) a_{2} f_{1} h_{2} \\
& \left.+\cos \left(2 \varphi_{1}\right) h_{6} a_{1} f_{1}\right)^{2} \\
& \left(k_{2} a_{2}\right)^{2}+\left(a_{2} \sigma_{2}-k_{6} a_{2}^{3}-k_{1} a_{2}-k_{5} a_{1}^{2}\right)^{2} \\
= & 4 a_{1}^{2}\left(\cos \varphi_{1}\right)^{2} k_{7} k_{4} f_{1}-2 a_{1}^{2} \sin \left(2 \varphi_{1}\right) k_{7} k_{3} f_{2}+k_{7}^{2} a_{1}^{4}-2 a_{1}^{2} k_{7} k_{4} f_{1}+k_{3}^{2}+f_{2}^{2}+k_{4}^{2} f_{1}^{2} .
\end{aligned}
$$

Let $\psi_{1}$ and $\phi_{1}$ in Eq. (13) be rewritten in the following forms:

$$
\begin{aligned}
& \psi_{1}=x_{1}+\mathrm{i} x_{2}, \\
& \phi_{1}=x_{3}+\mathrm{i} x_{4} .
\end{aligned}
$$

Substitute the above formula into Eq. (13). Then, the corresponding averaged equations in 
the Cartesian coordinates are given as follows:

$$
\begin{aligned}
\dot{x}_{1}= & \left(f_{2} h_{5}+h_{4}\right) x_{1}+\left(\sigma_{1}-h_{3}+f_{1} h_{6}\right) x_{2}+f_{2} h_{1} x_{3}-f_{1} h_{2} x_{4} \\
& -h_{8} x_{2}\left(x_{1}^{2}+x_{2}^{2}\right)-h_{9} x_{4}\left(x_{1}^{2}-x_{2}^{2}\right)-h_{7} x_{2}\left(x_{3}^{2}+x_{4}^{2}\right)+2 h_{9} x_{1} x_{2} x_{3}, \\
\dot{x}_{2}= & \left(h_{3}-\sigma_{1}+f_{1} h_{6}\right) x_{1}+\left(h_{4}-f_{2} h_{5}\right) x_{2}+f_{1} h_{2} x_{3}+f_{2} h_{1} x_{4}+h_{8} x_{1}\left(x_{1}^{2}+x_{2}^{2}\right) \\
& +h_{9} x_{3}\left(x_{1}^{2}-x_{2}^{2}\right)+h_{7} x_{1}\left(x_{3}^{2}+x_{4}^{2}\right)+2 h_{9} x_{1} x_{2} x_{4}, \\
\dot{x}_{3}= & f_{2} k_{3} x_{1}-f_{1} k_{4} x_{2}+k_{2} x_{3}+\left(\sigma_{2}-k_{1}\right) x_{4}-k_{7} x_{2}\left(3 x_{1}^{2}-x_{2}^{2}\right)-k_{5} x_{4}\left(x_{1}^{2}+x_{2}^{2}\right) \\
& -k_{6} x_{4}\left(x_{3}^{2}+x_{4}^{2}\right), \\
\dot{x}_{4}= & f_{1} k_{4} x_{1}+f_{2} k_{3} x_{2}+\left(k_{1}-\sigma_{2}\right) x_{3}+k_{2} x_{4}+k_{7} x_{1}\left(x_{1}^{2}-3 x_{2}^{2}\right)+k_{5} x_{3}\left(x_{1}^{2}+x_{2}^{2}\right) \\
& +k_{6} x_{3}\left(x_{3}^{2}+x_{4}^{2}\right) .
\end{aligned}
$$

\section{Numerical simulation}

\subsection{Numerical simulation of frequency-response}

The variation trends of the stiffness characteristic of the steady-state rotating cantilever blade enduring the aerodynamic force in the series form in the subsonic airflow are analyzed numerically in this section. Considering the operating condition of the compressor blade, it is obvious that the nonlinear responses of the blade can be affected by various factors, such as the linear parameters, the nonlinear parameters, and the aerodynamic force. Therefore, their effects on the frequency-responses for the plate are analyzed by numerical simulation.

Considering the weakly coupled condition, namely, let $a_{2}=1$ in Eq. (16a) and $a_{1}=1$ in Eq. (16b), the frequency-response curves of the cantilever blade structure for the first two order modes are obtained, as shown in Fig. 2, which illustrates that the plate performs hard spring properties. The effects of different factors on the frequency-responses of the cantilever plate are depicted in Fig. 3. Due to the nonlinearity, the multi-valued phenomenon is exhibited in Fig. 3.

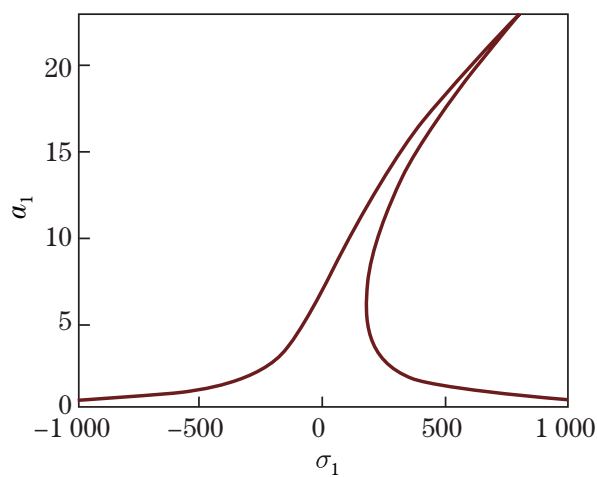

(a)

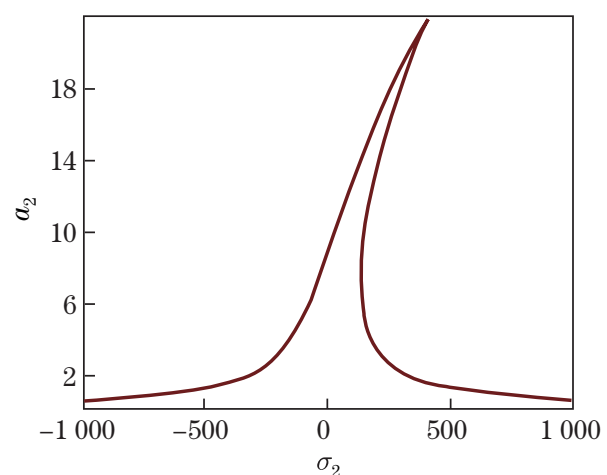

(b)

Fig. 2 Frequency-response curves: (a) uncoupled frequency-response curve of the 1st order mode; (b) uncoupled frequency-response curve of the 2nd order mode (color online)

Figures 3(a), 3(b), 3(e), and 3(f) show that, when the first harmonics of $f_{1}, h_{6}$, and $k_{3}$ increase, respectively, the nonlinear vibration amplitudes and the response bandwidths of the first two modes both increase. When the nonlinear parameters $\left|h_{8}\right|$ and $\left|k_{6}\right|$ increase, the dynamical response amplitudes decrease. Moreover, the larger $\left|h_{8}\right|$ and $\left|k_{6}\right|$ are, the greater the curvatures of the curves become, as shown in Figs. 3(c) and 3(d).

With the decrease in the frequencies $\sigma_{1}$ and $\sigma_{2}$, the frequency-response curves jump down to lower amplitudes for soft spring properties while jump up to higher amplitudes for hard spring properties. The signs of $h_{8}$ and $k_{6}$ can change the spring characteristics of the system. When the 


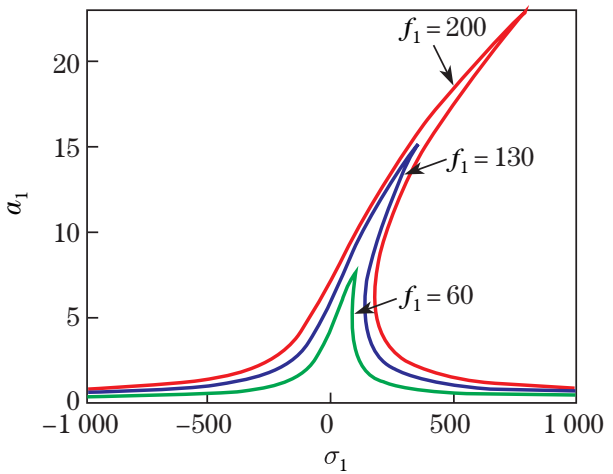

(a)

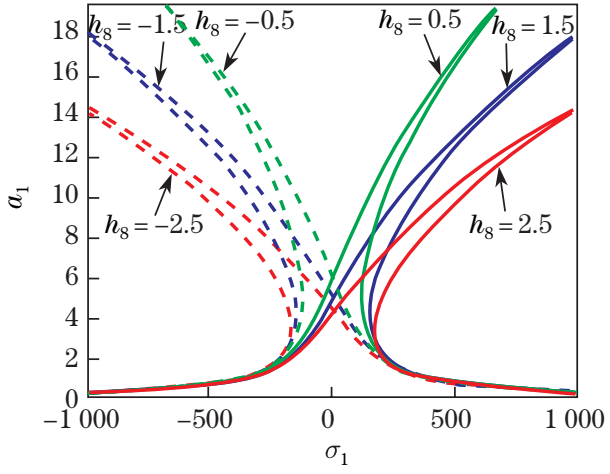

(c)

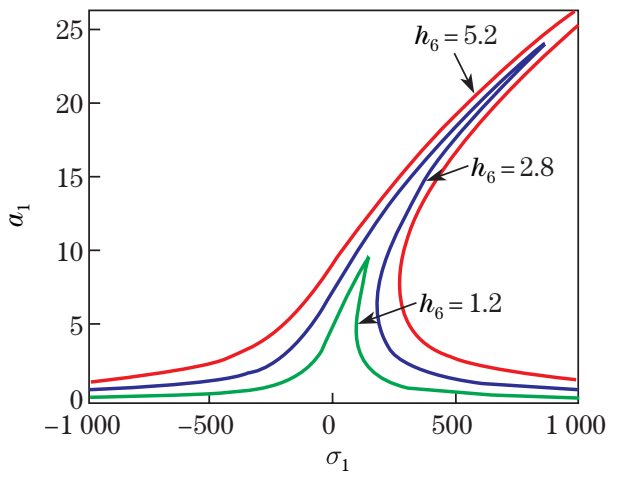

(e)

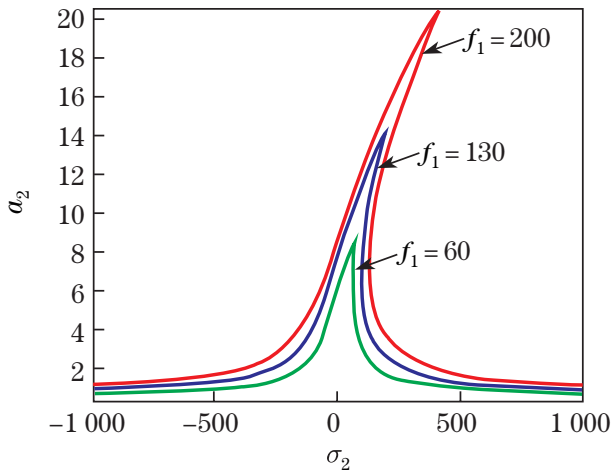

(b)

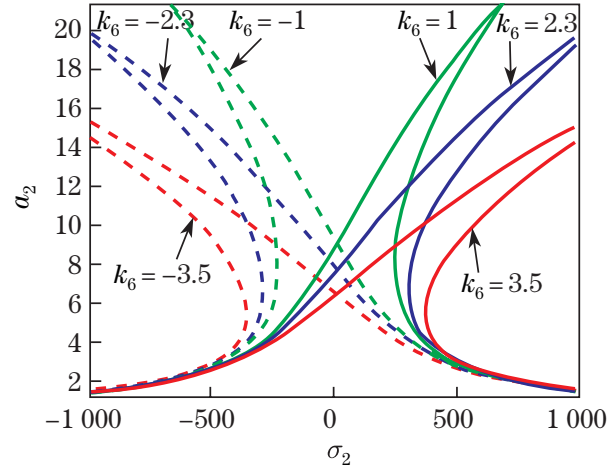

(d)

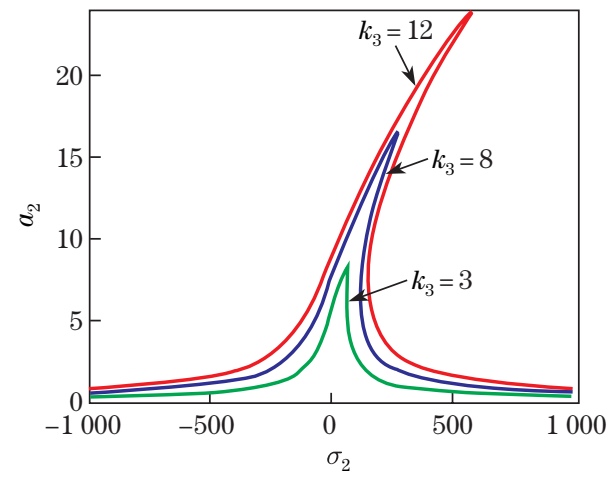

(f)

Fig. 3 Frequency-response curves for the cantilever rotating blade: (a) effects of the peak of the first harmonic of the aerodynamic force on frequency-response curves for the 1st order mode; (b) effects of the first harmonic of the aerodynamic force on frequency-response curves of the 2nd order mode; (c) effects of the nonlinear parameter of the dynamic equations of the blade on frequency-response curves of the 1st order mode; (d) effects of the nonlinear parameter of the dynamic equations of the blade on frequency-response curves of the 2nd order mode; (e) effects of the linear parameter on frequency-response curves of the 1st order mode; (f) effects of the linear parameter on frequency-response curves of the 2 nd order mode (color online)

nonlinear parameters $h_{8}$ and $k_{6}$ are positive, the system exhibits hard spring characteristics. However, when $h_{8}$ and $k_{6}$ are negative, the system has soft spring characteristics. When the signs of $h_{8}$ and $k_{6}$ are opposite, the corresponding curves are symmetrical about the vertical axis. 


\subsection{Numerical simulation of nonlinear behaviors}

Besides the steady-state analysis, the nonlinear dynamical behaviors of the compressor blade subject to the aerodynamic load and the centrifugal force are also numerically investigated based on Eq. (18). Except for the first harmonic of the aerodynamic force $f_{1}$ or $f_{2}$, keep other parameters and the initial condition unchanged so as to investigate the effects of the first harmonic of the aerodynamic force on nonlinear oscillations of the rotating plate. When $f_{1}$ or $f_{2}$ changes in a certain interval, bifurcation diagrams, in which complicated oscillation behaviors can be found, are numerically depicted based on the Poincaré map theory and RungeKutta algorithm. Unless otherwise stated, the parameters are set as $\sigma_{1}=1.3842, \sigma_{2}=1.2312$, $h_{1}=0.313, h_{2}=0.285, h_{3}=-1.127, h_{4}=-0.918, h_{5}=-0.049, h_{6}=-0.1833, h_{7}=0.151$, $h_{8}=0.0995, h_{9}=0.829, k_{1}=0.798, k_{2}=-0.964, k_{3}=0.0465, k_{4}=-1.4016, k_{5}=0.002$, $k_{6}=0.3293, k_{7}=0.525, f_{2}=5, x_{10}=0.3, x_{20}=-0.0101, x_{30}=-0.26$, and $x_{40}=0.2$, and $f_{1}$ varies in the interval of $f_{1} \in[0,4]$. Thus, Fig. 4 is obtained.

Figures 4(a) and 4(b) describe the bifurcation diagrams about the first harmonic of the aerodynamic force $f_{1}$ to the lateral displacements $x_{1}$ and $x_{3}$, respectively, when $f_{1}$ varies in the interval of $f_{1} \in[0,4]$. Select $f_{2}$ as the control parameter to detect the impact of $f_{2}$ on the nonlinear vibrations of the rotating blade. Figures 5(a) and 5(b) describe the bifurcations of $f_{2}$ to the lateral displacements $x_{1}$ and $x_{3}$ when $f_{1}$ is chosen as $f_{1}=4$, respectively. It can be observed from Figs. 4 and 5 that chaotic and periodic motions occur in the vibration of the blade. The diagrams show that the structure is sensitive to the first harmonics of the aerodynamic forces $f_{1}$ and $f_{2}$. Figures 4 and 5 illustrate that, with the increase in the first harmonic of the aerodynamic force, the blade performs complex dynamical behaviors.

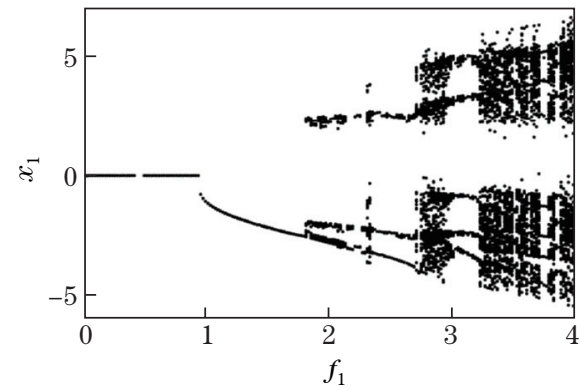

(a)

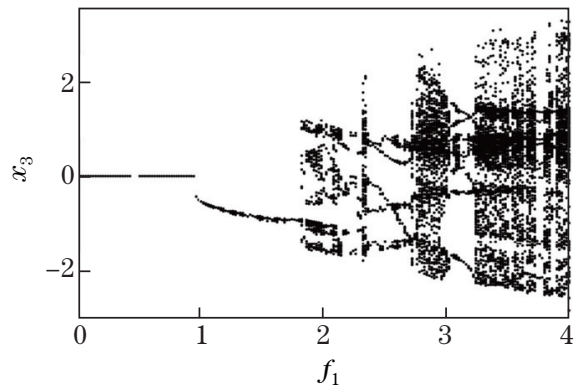

(b)

Fig. 4 Bifurcation diagrams for $f_{1} \in[0,4]$ about the first harmonic of the aerodynamic force $f_{1}$ : (a) bifurcation diagram about $f_{1}$ to the 1st lateral displacement $x_{1}$; (b) bifurcation diagram about $f_{1}$ to the 2 nd lateral displacement $x_{3}$

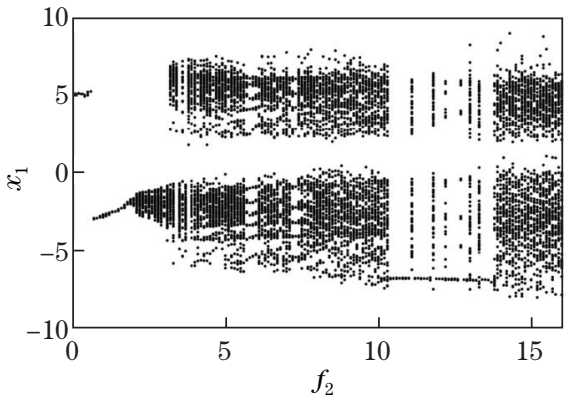

(a)

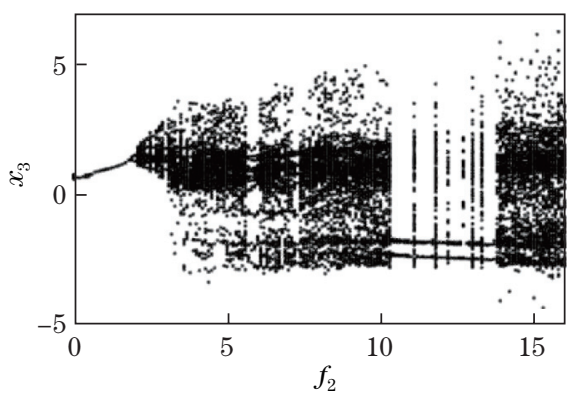

(b)

Fig. 5 Bifurcation diagrams for $f_{2} \in[0,16]$ about the first harmonic of the aerodynamic force $f_{2}$ : (a) bifurcation diagram about $f_{2}$ to the 1st lateral displacement $x_{1}$; (b) bifurcation diagram about $f_{2}$ to the 2 nd lateral displacement $x_{3}$ 
The chaotic and periodic responses can be identified by several conventional criteria. Thus, the bifurcation diagrams, waveforms, phase portraits, and power spectra are utilized to further verify the existence of the chaotic and periodic motions of the blade. Figure 6 illustrates that the blade performs period- 1 motion as the first harmonic of the aerodynamic force $f_{1}$ is chosen as $f_{1}=0.9$. Figure 7 shows that the system exhibits period- 3 motion as the first harmonic of the aerodynamic force $f_{1}$ is chosen as $f_{1}=1.98$. The bifurcation diagrams in Fig. 4 demonstrate that, as $f_{1}$ increases, the multi-periodic motion of the blade changes with the chaotic motion. Due to the limited space, only two diagrams are depicted here. When the first harmonic of

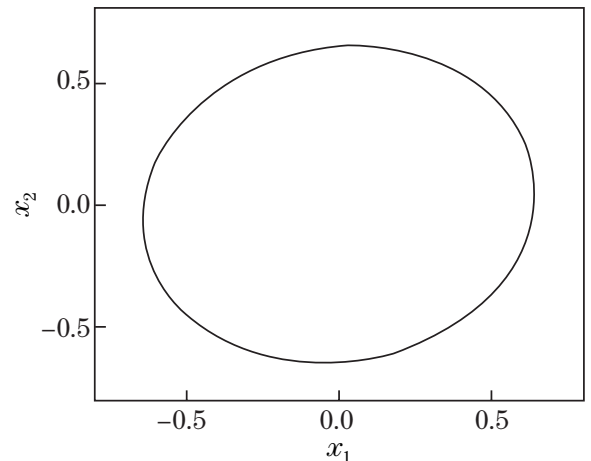

(a)

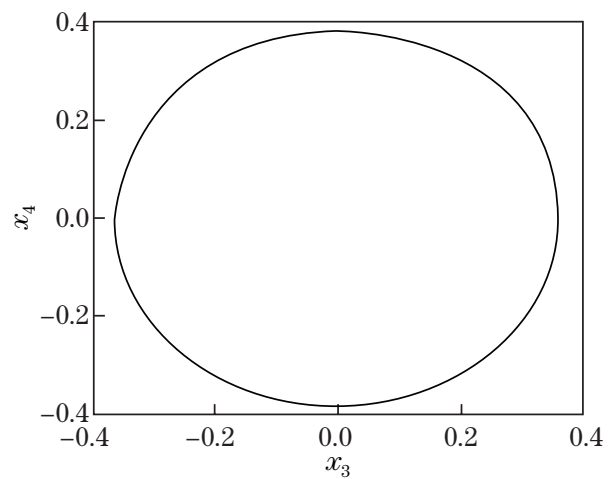

(c)

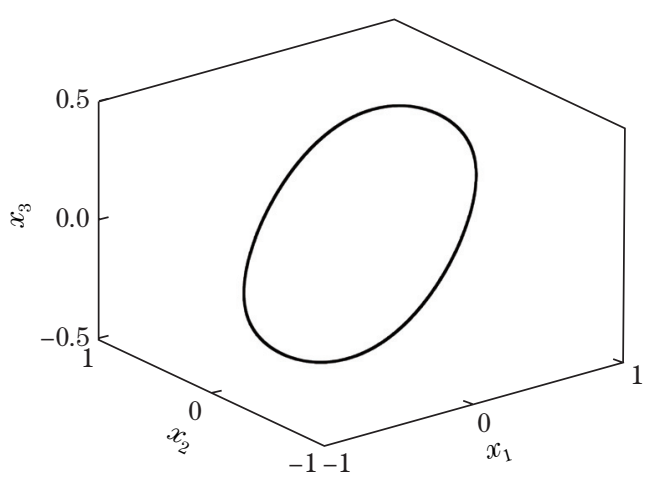

(e)

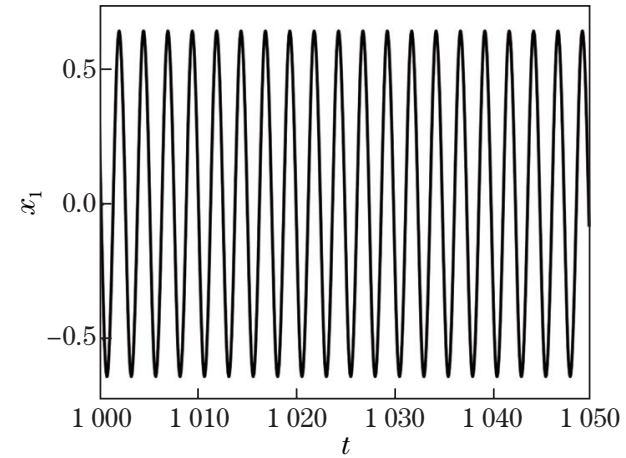

(b)

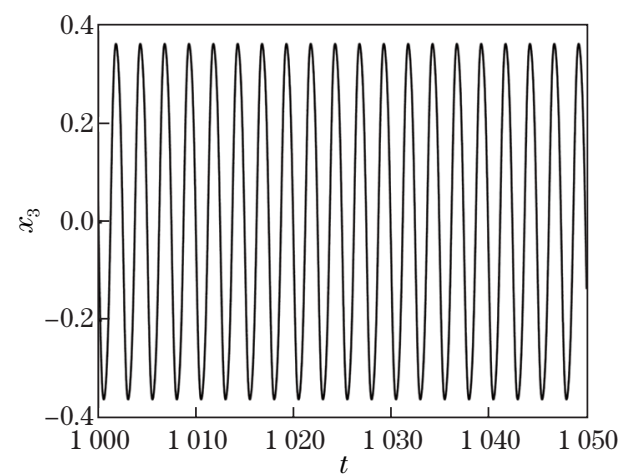

(d)

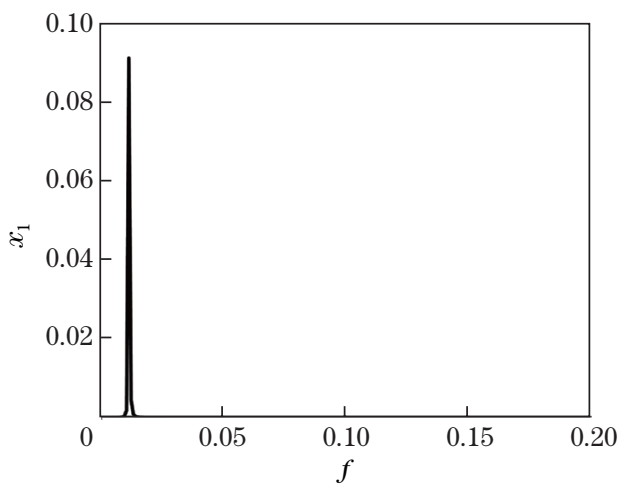

(f)

Fig. 6 The period- 1 motion of the blade when $f_{1}=0.9$ : (a) the phase portrait in the $x_{1} x_{2}$-plane; (b) the waveform for $x_{1}$; (c) the phase portrait in the $x_{3} x_{4}$-plane; (d) the waveform for $x_{3}$; (e) the three-dimensional phase portrait; (f) the power spectrum 
the aerodynamic $f_{1}$ is changed as $f_{1}=3$, the system performs multi-periodic motion, as shown in Fig. 8. Figure 9 exhibits the chaotic motion of the system when the parameter $f_{1}$ is selected as $f_{1}=3.91$. It can be seen in Fig. 5 that the multi-periodic motion of the plate changes with its chaotic motion when $f_{2}$ is larger than 2.6. When $f_{2}$ is chosen as $f_{2}=2.6$, the system exhibits the multi-periodic motion, as shown in Fig. 10. When $f_{2}$ increases to 15 , the system performs chaotic motion, as shown in Fig. 11.

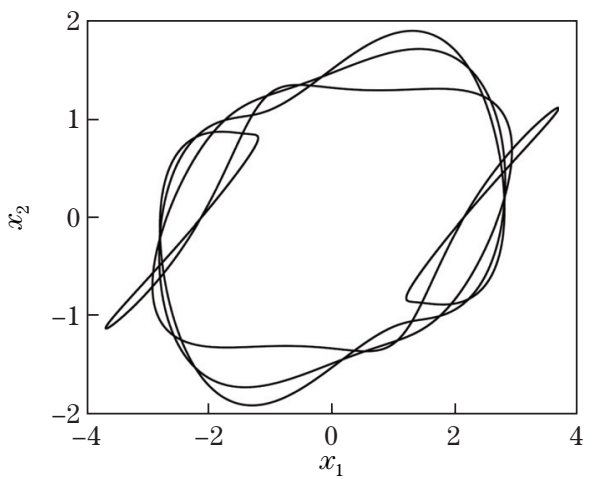

(a)

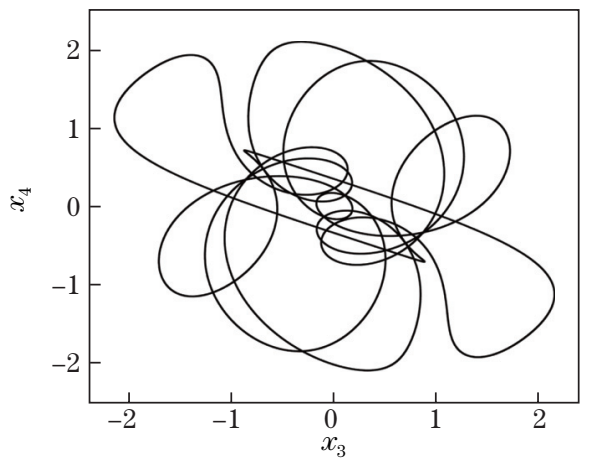

(c)

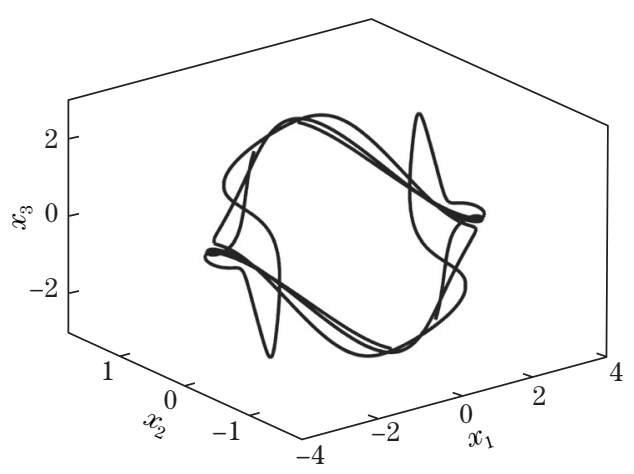

(e)

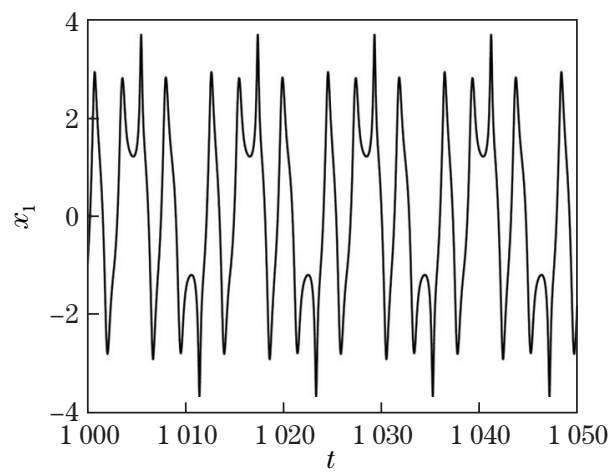

(b)

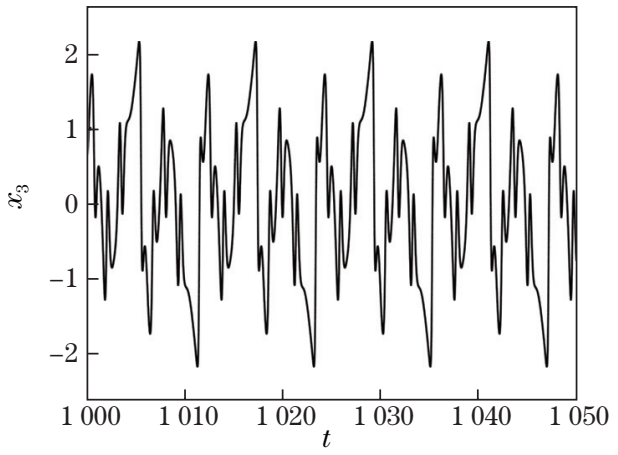

(d)

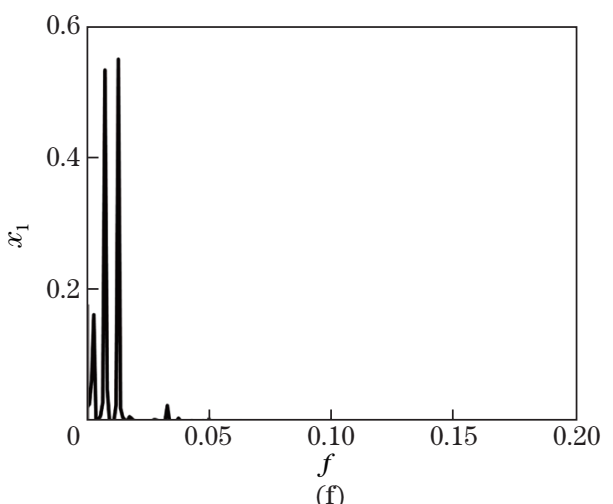

Fig. 7 The period-3 motion of the blade when $f_{1}=1.98$ : (a) the phase portrait in the $x_{1} x_{2}$-plane; (b) the waveform for $x_{1}$; (c) the phase portrait in the $x_{3} x_{4}$-plane; (d) the waveform for $x_{3}$; (e) the three-dimensional phase portrait; (f) the power spectrum

According to the numerical simulation, the system performs complex dynamic behaviors when the parameters $f_{1}$ and $f_{2}$ change in a certain region. The first harmonic of the aerodynamic force significantly affects the dynamical vibrations of the blade. 


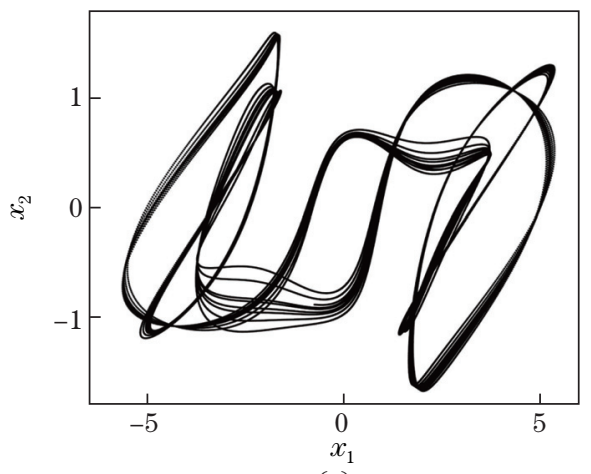

(a)

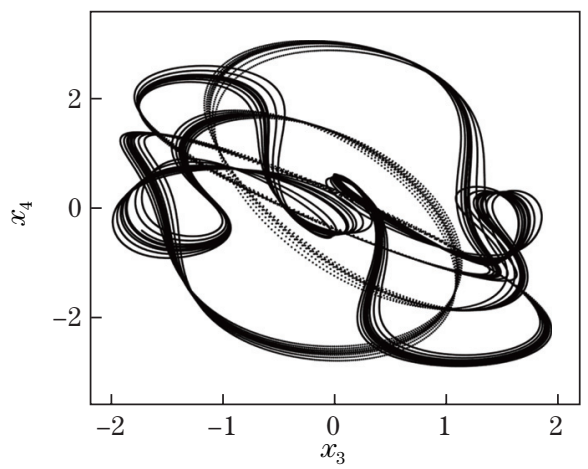

(c)

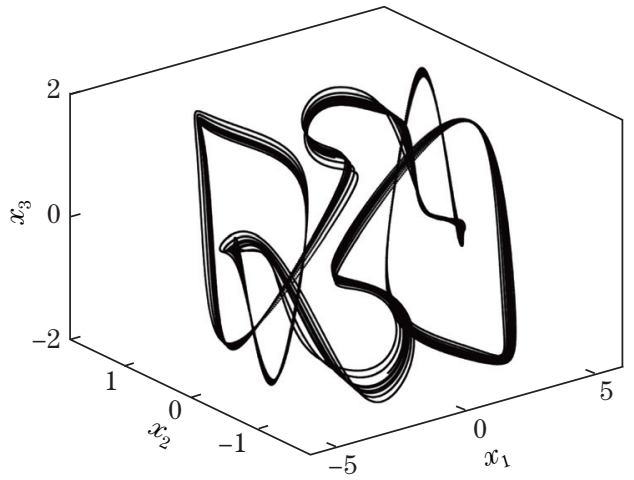

(e)

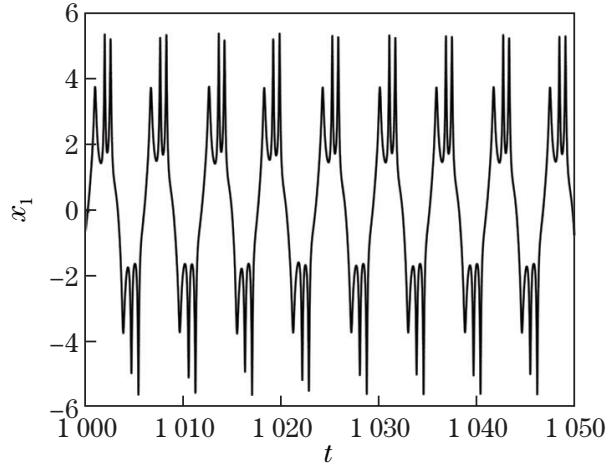

(b)

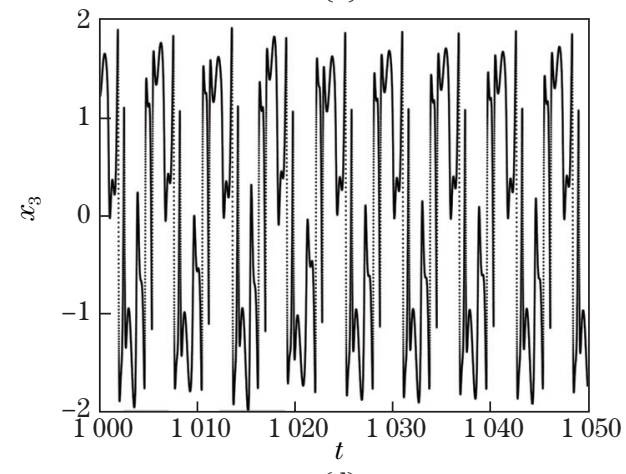

(d)

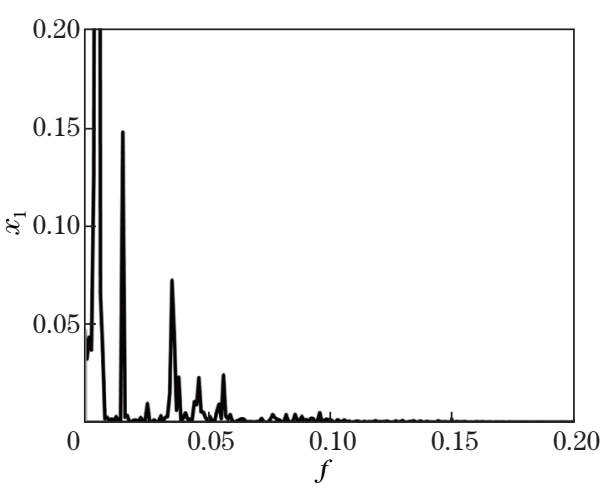

(f)

Fig. 8 The multi-periodic motion of the blade when $f_{1}=3$ : (a) the phase portrait in the $x_{1} x_{2}$-plane; (b) the waveform for $x_{1}$; (c) the phase portrait in the $x_{3} x_{4}$-plane; (d) the waveform for $x_{3}$; (e) the three-dimensional phase portrait; (f) the power spectrum

\section{Conclusions}

In this paper, the traditional piston theory is not used. The nonlinear vibration of the plate rotating with a constant speed subject to the aerodynamic load in series form is studied. The nonlinear partial differential governing equations of the rotating blade are derived by using Hamilton's principle. Ordinary differential equations are obtained by discretizing the nonlinear partial differential governing equations with the Galerkin method. Asymptotic perturbation methodology is used to obtain the averaged equation for $1: 3$ internal resonance and $1 / 2$ subharmonic resonance rotating blade. The nonlinear dynamics and frequency-responses for the blade are investigated numerically. 


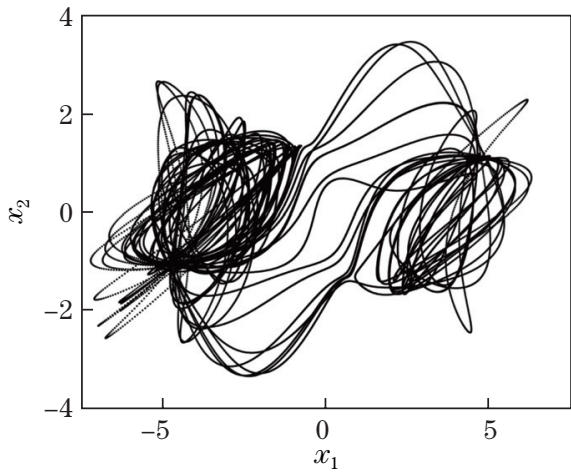

(a)

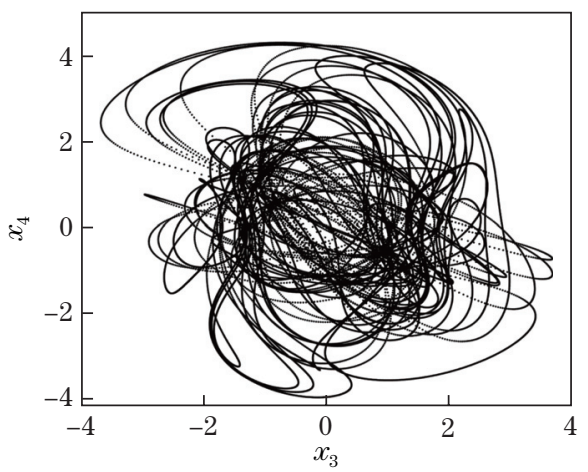

(c)

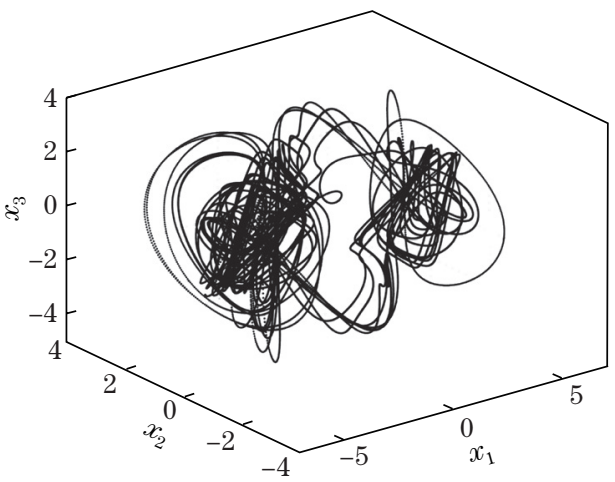

(e)

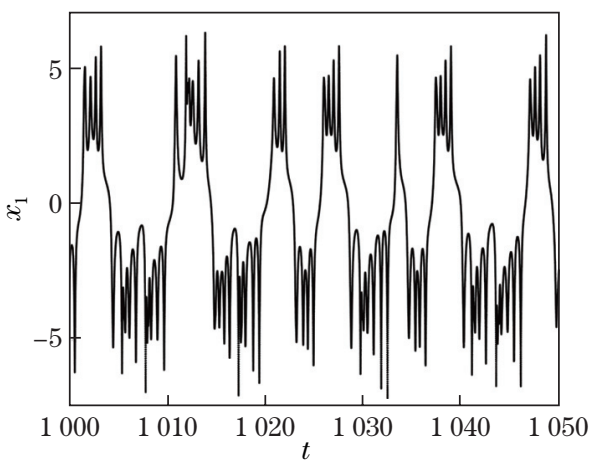

(b)

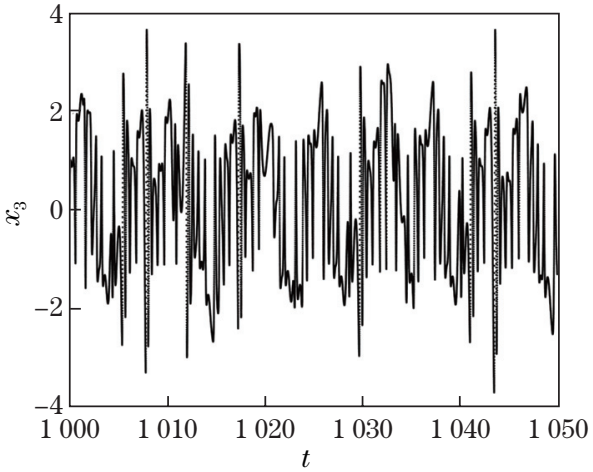

(d)

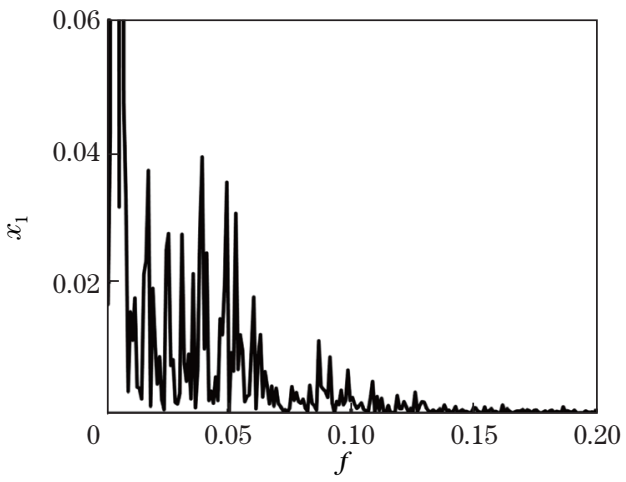

(f)

Fig. 9 The chaotic motion of the blade when $f_{1}=3.91$ : (a) the phase portrait in the $x_{1} x_{2}$-plane; (b) the waveform for $x_{1}$; (c) the phase portrait in the $x_{3} x_{4}$-plane; (d) the waveform for $x_{3}$; (e) the three-dimensional phase portrait; (f) the power spectrum

Jumping of the solutions is described clearly in frequency-response curves. The effects of various factors on the frequency-response curves show that the system spring property is determined by whether the certain nonlinear parameter is larger or smaller than zero. The increase in the aerodynamic force and linear parameters results in the existence of the hard spring characteristic of the blade. Meanwhile, the increase in the aerodynamic force and linear parameters causes the increase in the vibration amplitudes and the response bandwidth of the first two modes. However, when the nonlinear parameters increase, the amplitude of the dynamic responses decreases.

Based on Eq. (18), bifurcation diagrams, phase portraits, waveforms, and power spectra are obtained to demonstrate that periodic motions and chaotic motions occur in the nonlinear 


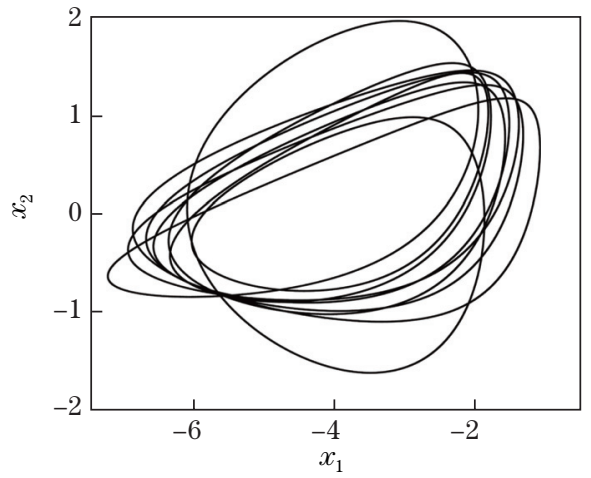

(a)

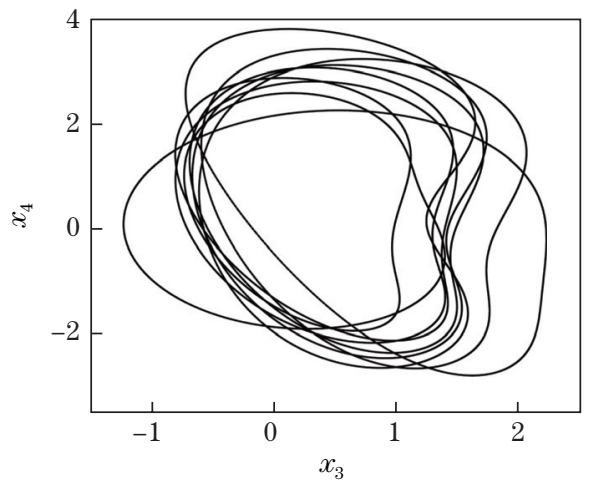

(c)

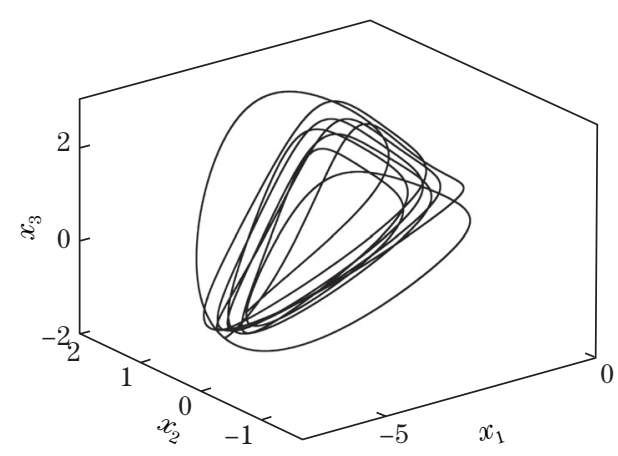

(e)

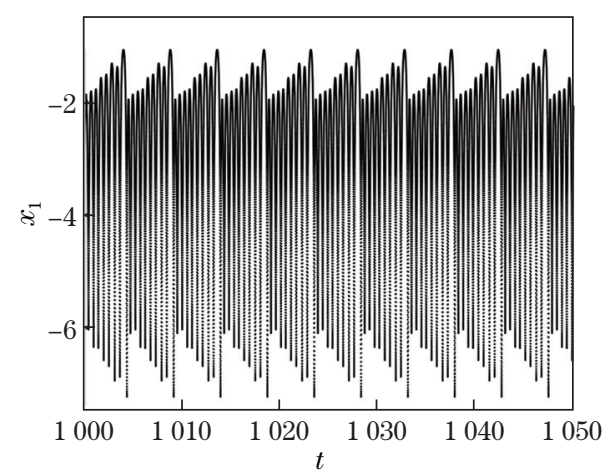

(b)

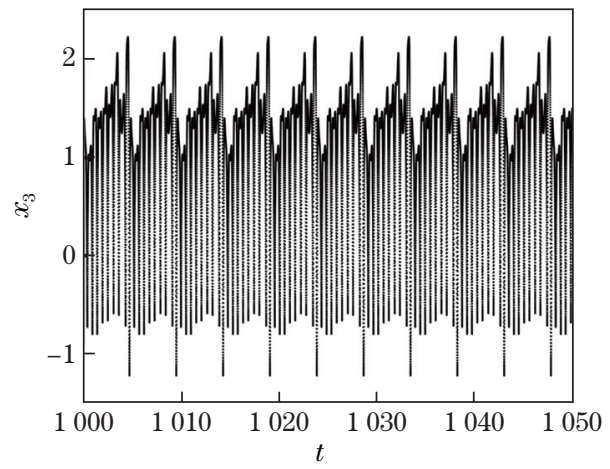

(d)

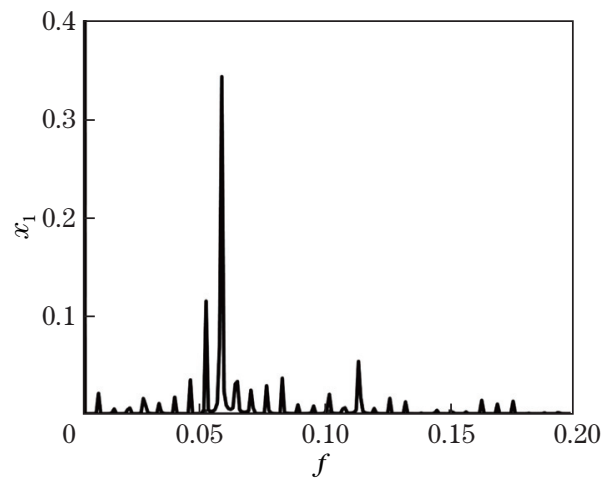

(f)

Fig. 10 The multi-periodic motion of the blade when $f_{2}=2.6$ : (a) the phase portrait in the $x_{1} x_{2}$ plane; (b) the waveform for $x_{1}$; (c) the phase portrait in the $x_{3} x_{4}$-plane; (d) the waveform for $x_{3}$; (e) the three-dimensional phase portrait; (f) the power spectrum

vibrations of the rotating blade under certain conditions. The bifurcation diagrams, phase portraits, waveforms, and power spectra numerically depicted demonstrate that system nonlinear oscillations can be easily affected by the first harmonic of the aerodynamic force. Period-1 motion, period-3 motion, multi-periodic motion, and chaotic motion occur when $f_{1}$ and $f_{2}$ are chosen in specified intervals. From mathematical points of view, in the certain initial condition, the appearance of amplitude-modulated periodic and chaotic vibrations for original systems is explained by the bifurcation diagrams that we have obtained. The large-amplitude vibrations of the blade with a constant rotating speed that may lead to undesirable damage of the aero-engine can be controlled by adjusting the aerodynamic force. Therefore, it is of great significance to investigate the nonlinear behaviors of blades to reduce the probability of aero-engine failure. 


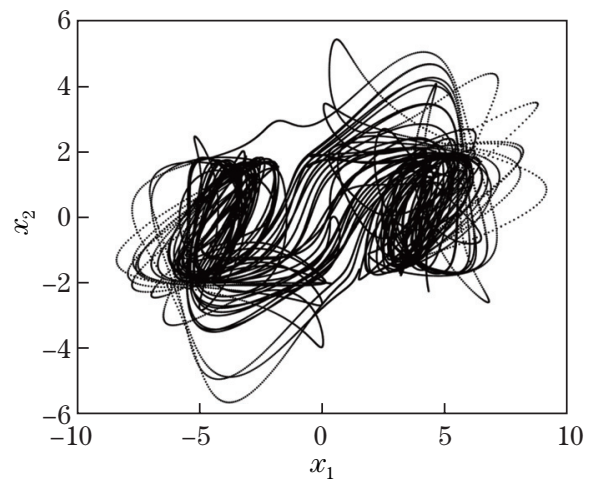

(a)

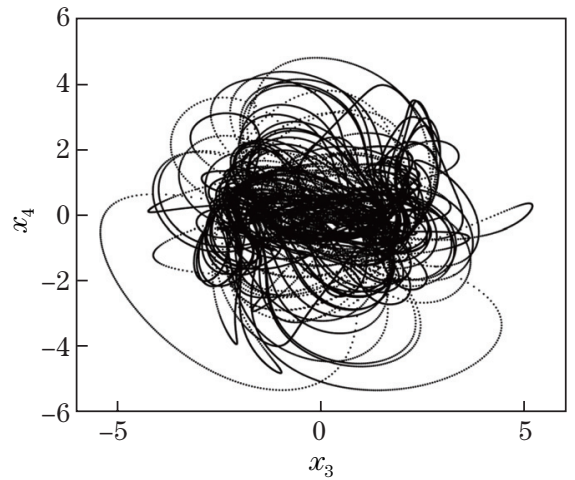

(c)

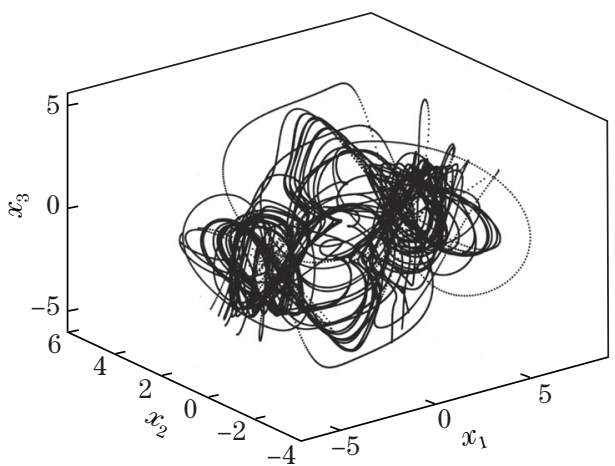

(e)

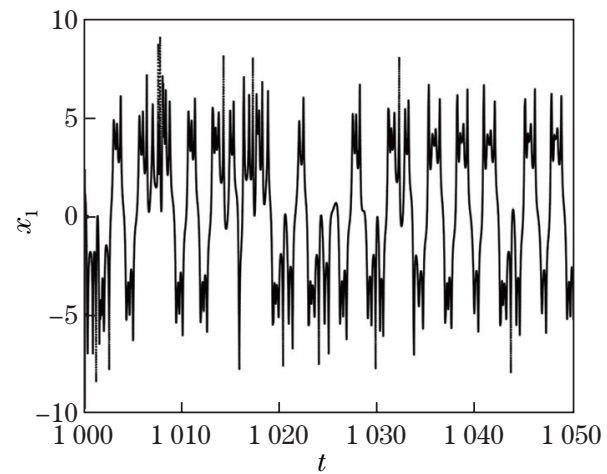

(b)

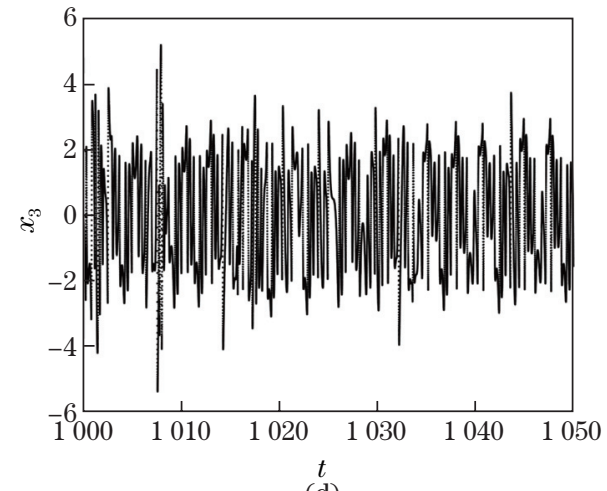

(d)

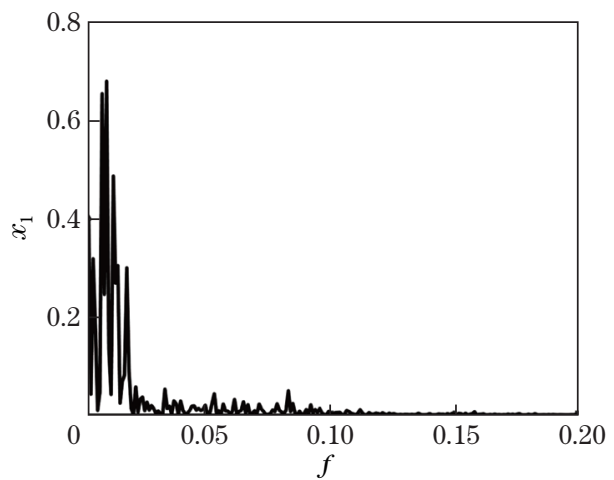

(f)

Fig. 11 The chaotic motion of the blade when $f_{2}=15$ : (a) the phase portrait in the $x_{1} x_{2}$-plane; (b) the waveform for $x_{1}$; (c) the phase portrait in the $x_{3} x_{4}$-plane; (d) the waveform for $x_{3}$; (e) the three-dimensional phase portrait; (f) the power spectrum

Open Access This article is licensed under a Creative Commons Attribution 4.0 International License, which permits use, sharing, adaptation, distribution and reproduction in any medium or format, as long as you give appropriate credit to the original author(s) and the source, provide a link to the Creative Commons licence, and indicate if changes were made. To view a copy of this licence, visit http://creativecommons.org/licenses/by/4.0/.

\section{References}

[1] MA, H., XIE, F. T., NAI, H. Q., and WEN, B. C. Vibration characteristics analysis of rotating shrouded blades with impacts. Journal of Sound and Vibration, 378, 92-108 (2016) 
[2] DING, H., ZHU, M. H., and CHEN, L. Q. Dynamic stiffness method for free vibration of an axially moving beam with generalized boundary conditions. Applied Mathematics and Mechanics (English Edition), 40, 911-924 (2019) https://doi.org/10.1007/s10483-019-2493-8

[3] YANG, X. D., WANG, S. W., ZHANG, W., QIN, Z. H., and YANG, T. Z. Dynamic analysis of a rotating tapered cantilever Timoshenko beam based on the power series method. Applied Mathematics and Mechanics (English Edition), 38(10), 1425-1438 (2017) https://doi.org/ 10.1007/s10483-017-2249-6

[4] SABUNCU, M. and THOMAS, J. Vibration characteristics of pretwisted aerofoil cross-section blade packets under rotating conditions. AIAA Journal, 30, 241-250 (2015)

[5] LI, L. and ZHANG, D. G. Free vibration analysis of rotating functionally graded rectangular plates. Composites Structures, 136, 493-504 (2015)

[6] MAHI, A., BEDIA, E. A., and TOUNSI, A. A new hyperbolic shear deformation theory for bending and free vibration analysis of isotropic, functionally graded, sandwich and laminated composite plates. Applied Mathematical Modelling, 39(9), 2489-2508 (2015)

[7] BENNOUN, M., HOUARI, M. S. A., and TOUNSI, A. A novel five-variable refined plate theory for vibration analysis of functionally graded sandwich plates. Mechanics of Advanced Materials and Structures, 23(4), 423-431 (2016)

[8] XIE, J. S., ZI, Y. Y., ZHANG, M. Q., and LUO, Q. Y. A novel vibration modeling method for a rotating blade with breathing cracks. Science China Technological Sciences, 62, 333-348 (2019)

[9] NIU, Y., ZHANG, W., and GUO, X. Y. Free vibration of rotating pretwisted functionally graded composite cylindrical panel reinforced with graphene platelets. European Journal of MechanicsA/Solids, 77, 103798 (2019)

[10] ZHANG, W. and ZHAO, M. H. Nonlinear vibrations of a composite laminated cantilever rectangular plate with one-to-one internal resonance. Nonlinear Dynamics, 70, 295-313 (2012)

[11] CHEN, L. Q., ZHANG, Y. L., ZHANG, G. C., and DING, H. Evolution of the double-jumping in pipes conveying fluid flowing at the supercritical speed. International Journal of Non-Linear Mechanics, 58, 11-21 (2014)

[12] SUBRAhMANYAM, K. B., KAZA, K. R. V., BROWN, G. V., and LAWRENCE, C. Nonlinear vibration and stability of rotating, pretwisted, preconed blades including coriolis effects. Journal of Aircraft, 24(5), 342-352 (2012)

[13] YAO, M. H., ZHANG, W., and CHEN, Y. P. Analysis on nonlinear oscillations and resonant responses of a compressor blade. Acta Mechanica, 225, 3483-3510 (2014)

[14] ROY, P. A. and MEGUID, S. A. Nonlinear transient dynamic response of a blade subject to a pulsating load in a decaying centrifugal force field. International Journal of Mechanics and Materials in Design, 14, 709-728 (2018)

[15] WANG, D., CHEN, Y. S., WIERCIGROCH, M., and CAO, Q. J. Bifurcation and dynamic response analysis of rotating blade excited by upstream vortices. Applied Mathematics and Mechanics (English Edition), 37(9), 1251-1274 (2016) https://doi.org/10.1007/s10483-016-2128-6

[16] ZHOU, Y. and ZHANG, W. Double Hopf bifurcation of composite laminated piezoelectric plate subjected to external and internal excitations. Applied Mathematics and Mechanics (English Edition), 38(5), 689-706 (2017) https://doi.org/10.1007/s10483-017-2196-9

[17] LI, S. B. and ZHANG, W. Global bifurcations and multi-pulse chaotic dynamics of rectangular thin plate with one-to-one internal resonance. Applied Mathematics and Mechanics (English Edition), 33(9), 1115-1128 (2012) https://doi.org/10.1007/s10483-012-1609-9

[18] ZHANG, B., DING, H., and CHEN, L. Q. Super-harmonic resonances of a rotating pre-deformed blade subjected to gas pressure. Nonlinear Dynamics, 98, 2531-2549 (2019)

[19] ZHANG, B., DING, H., and CHEN, L. Q. Subharmonic and combination resonance of rotating pre-deformed blades subjected to high gas pressure. Acta Mechanica Solida Sinica (2020) https://doi.org/10.1007/s10338-020-00168-X

[20] DING, H., HUANG, L. L., MAO, X. Y., and CHEN, L. Q. Primary resonance of traveling viscoelastic beam under internal resonance. Applied Mathematics and Mechanics (English Edition), 38(1), 1-14 (2017) https://doi.org/10.1007/s10483-016-2152-6 
[21] LIU, Y. Z., HAO, Y. X., ZHANG, W., CHEN, J., and LI, S. B. Nonlinear dynamics of initially imperfect functionally graded circular cylindrical shell under complex loads. Journal of Sound and Vibration, 348, 294-328 (2015)

[22] YAO, M. H., NIU, Y., and HAO, Y. X. Nonlinear dynamic responses of rotating pretwisted cylindrical shells. Nonlinear Dynamics, 95, 151-174 (2019)

[23] YAO, M. H., MA, L., ZHANG, M. M., and ZHANG, W. Vibration characteristics analysis of the rotating blade based on an polynomial aerodynamic force. Proceedings of the ASME 2017 International Design Engineering Technical Conferences and Computers and Information in Engineering Conference, ASME, Ohio, USA (2017)

[24] ZHANG, J., XU, J., YUAN, X. G., DING, H., NIU, D. T., and ZHANG, W. Z. Nonlinear vibration analyses of cylindrical shells composed of hyperelastic materials. Acta Mechanica Solida Sinica, 32(4), 463-482 (2019)

[25] DING, H., LI, Y., and CHEN, L. Q. Effects of rotary inertia on sub- and super-critical free vibration of an axially moving beam. Meccanica, 53(13), 3233-3249 (2018)

[26] HAO, Y. X., CHEN, L. H., ZHANG, W., and LEI, J. G. Nonlinear oscillations, bifurcations and chaos of functionally graded materials plate. Journal of Sound and Vibration, 312, 862-892 (2008) 